Publ. RIMS, Kyoto Univ.

13 (1977), 589-626

\title{
On the Long-Range Stationary Wave Operator
}

\author{
By \\ Hiroshi ISOZAKI*

\section{$\S 0$. Introduction}

In the present paper we shall be concerned with the stationary theory of scattering associated with the Schrödinger operator with a long-range potential.

In quantum theory of scattering, many authors have investigated the existence and completeness of wave operators $W^{ \pm}=\mathrm{s}_{t \rightarrow \pm \infty} \lim ^{i t H_{2}} e^{-i t H_{1}}$, where $H_{1}$ and $H_{2}$ are self-adjoint operators acting on a Hilbert space $\mathcal{H}$. Among them, Kato and Kuroda gave an abstract time-independent approach to scattering theory. They derived a stationary form of the wave operator in the following way:

$$
W^{ \pm}=\int_{-\infty}^{\infty} E_{2}^{\prime}(\lambda)\left(H_{2}-(\lambda \pm i 0)\right) R_{1}(\lambda \pm i 0) P_{1} d \lambda
$$

where $E_{2}(\lambda)$ denotes the resolution of the identity for $H_{2}, E_{2}^{\prime}(\lambda)$ is the "formal" derivative of $E_{2}(\lambda)$, and $R_{1}(z)$ and $P_{1}$ denote the resolvent and the projection onto the absolutely continuous subspace of $H_{1}$, respectively. They discussed in their abstract theory the existence and unitary property of this operator and coincidence with the time-dependent one ([8], [9]). In the case of the Schrödinger operators, $H_{1}=-\Delta, H_{2}=-\Delta$ $+V(x)$, their theory covers general short-range potentials: i.e. $V(x)$ $=O\left(|x|^{-1-\varepsilon}\right)$ as $|x| \rightarrow \infty, \varepsilon>0$. But when $V(x)$ is a long-range potential $V(x)=O\left(|x|^{-\delta}\right), 0<\delta \leq 1$, the operator defined by (0.1) does not exist.

Recently, Pinchuk [10] has derived an appropriate modification of (0.1) in the case of a long-range potential. His remedy consists in inserting a unitary operator $U(\lambda \pm i 0)$ which depends on the concrete potential as follows:

Communicated by S. Matsuura, October 12, 1976.

* Department of Mathematics, Kyoto Univ., Kyoto, 606 Japan. 


$$
W^{ \pm}=\int_{-\infty}^{\infty} E_{2}^{\prime}(\lambda)\left(H_{2}-(\lambda \pm i 0)\right) U(\lambda \pm i 0) R_{1}(\lambda \pm i 0) P_{1} d \lambda .
$$

Using this form, he discussed the existence and completeness of the stationary wave operators for the various potentials. His decay assumptions on $V(x)$ are as follows:

$$
\begin{aligned}
& V(x)=V_{1}(x)+V_{2}(x), \\
& V_{1}(x), \Lambda V_{1}(x)=O\left(|x|^{-\varepsilon}\right), \\
& \frac{\partial}{\partial r} V_{1}(x), V_{2}(x)=O\left(|x|^{-1-\varepsilon}\right), \\
& \operatorname{grad}_{\omega} V_{1}(x)=O\left(|x|^{-3 / 2-\varepsilon}\right) \quad \text { as } \quad|x| \rightarrow \infty, \varepsilon>0,
\end{aligned}
$$

where $\operatorname{grad}_{\omega}=\operatorname{grad}-\omega \frac{\partial}{\partial r} \quad(\omega=x /|x|)$, and $\Lambda$ denotes the LaplaceBeltrami operator on the unit sphere. His choice for $U(\lambda \pm i 0)$ is the operator of multiplication by a function $\exp \left(-\frac{i}{2 \sqrt{ }} \bar{\lambda} \int_{0}^{|v|} V_{1}(s \omega) d s\right)$ and the method of construction is based upon Kato-Kuroda's abstract theory (especially upon the "spectral form").

The purpose of this paper is, influenced by the work of Pinchuk, to construct the stationary wave operator in the form of (0.2) for the general long-range potentials, and to discuss the unitary property. Our assumption on $V(x)$ is as follows:

$V(x)$ is a real $C^{m}$-function ( $m$ will be given precisely later in $\S 2)$, and $D^{k} V(x)=O\left(|x|^{-k-\delta}\right)$ as $|x| \rightarrow \infty, \delta>0, k \geq 0$, where $D^{k}$ denotes an arbitrary derivative of $k$-th order.

And our choice for $U(\lambda \pm i 0)$ is the operator of multiplication by the function $\exp (-i X(x, \sqrt{ } \lambda \pm i 0))$, where $X\left(x, \kappa_{1}\right)$ is an approximate solution of the non-linear equation $2 \kappa_{1} \frac{\partial X}{\partial r}=V(x)+|\nabla X|^{2}$.

Here we must mention the recent work of Saito [12] concerning the eigenfunction expansion associated with $\mathrm{H}_{2}$. He obtained the spectral representation of $\mathrm{H}_{2}$ in the following way: Define

$$
\mathscr{I}(\lambda) f=\pi^{-1 / 2} \lambda^{1 / 4} \operatorname{sim}_{r \rightarrow \infty} r^{(n-1) / 2} \exp (-i \sqrt{\lambda} r+i X(r \cdot)) R_{2}(\lambda+i 0) f(r \cdot)
$$

in $L_{2}\left(S^{n-1}\right)$, and set $(\mathscr{F} f)(\lambda)=\mathscr{F}(\lambda) f$. Then the operator $\mathscr{L}: \mathscr{H} \rightarrow$ $L_{2}\left((0, \infty): L_{2}\left(S^{n-1}\right)\right)$ gives the generalized Fourier transform associated 
with $H_{2}$. Here, $X$ is an approximate solution of equation $2 \sqrt{\lambda} \frac{\partial X}{\partial r}$ $=V(x)+|\nabla X|^{2}$. Our choice of $X$ is suggested by this work. And we can also clarify the relation between our stationary wave operator and Saitō's eigenfunction expansion theory.

The plan of this paper is as follows. In $\S 1$, we construct the stationary wave operator in a rather abstract way, but differing from Pinchuk, we do not use Kato-Kuroda's abstract theory. Some calculation lemmas needed for the application of the abstract theory are proved in $\S 2$. Our main theorem appears in $\S 3$. In $\S 4$, we discuss the coincidence of our stationary wave operator with the one obtained by the eigenfunction expansion theory. We shall give some remarks for the shortrange perturbation of our theory in $\S 5$. In the Appendix, we shall establish some a-priori estimates which play a crucial role in our context.

\section{$\S 1$. Construction of the Stationary Wave Operator}

In this section we construct the stationary wave operator in a rather abstract way. The author owes most of the ideas to Ikebe [6].

First we introduce some notations.

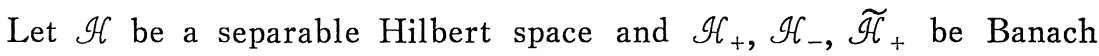
spaces. We assume the following inclusion relations for these spaces:

$$
\widetilde{\mathcal{H}}_{+} \subset \mathscr{H}_{+} \subset \mathscr{H}_{\mathcal{H}} \subset \mathscr{H}_{-},
$$

where all inclusions are dense and continuous. And moreover, we assume that $\mathscr{H}_{-}$is identified with the dual space of $\mathscr{H}_{+}$. We use (, ) to denote not only the inner product of $\mathscr{H}$ but also the coupling of $\mathscr{H}_{+}$ and $\mathscr{H}_{-}$, which will not confuse our argument. $\boldsymbol{C}$ and $\boldsymbol{R}$ denote the totality of complex and real numbers, respectively.

Let us consider two self-adjoint operators $H_{1}$ and $H_{2}$ on $\mathcal{H}$. We denote the resolvent of $H_{j}$ as follows $(j=1,2)$ :

$$
R_{j}(z)=\left(H_{j}-z\right)^{-1} \quad(z \in \boldsymbol{C}-\boldsymbol{R}) .
$$

The resolution of the identity for $H_{j}$ is denoted by $E_{j}(\lambda) \quad(j=1,2)$. In general, $\boldsymbol{B}\left(\mathscr{A}_{1}: \mathscr{A}_{2}\right)$ denotes the totality of bounded linear operators from a Banach space $\mathscr{A}_{1}$ into a Banach space $\dot{A}_{2}$. 
Now, we assume as follows.

(A-1) The limiting absorption principle is guaranteed. That $i s$, for arbitrary $\lambda>0, \varepsilon>0, R_{j}(\lambda \pm i \varepsilon) \in \boldsymbol{B}\left(\mathscr{H}_{+}: \mathscr{H}_{-}\right)$, and when $\varepsilon$ tends to 0 , there exists a strong limit $\mathrm{s}-\lim _{\varepsilon \rightarrow 0} R_{j}(\lambda \pm i \varepsilon) \equiv R_{j}(\lambda \pm i 0)$ $\in \boldsymbol{B}\left(\mathcal{H}_{+}: \mathcal{H}_{-}\right)$. Moreover for an arbitrary $f \in \mathcal{H}_{+}, R_{j}(\lambda \pm i 0) f$ is an $\mathscr{H}_{-}$-valucd strongly continuous function of $\lambda \quad(0<\lambda<\infty)$ $(j=1,2)$.

With the aid of this assumption we define for $j=1,2, \lambda>0$,

$$
E_{j}^{\prime}(\lambda)=\frac{1}{2 \pi i}\left(R_{j}(\lambda+i 0)-R_{j}(\lambda-i 0)\right) .
$$

This is a bounded linear operator from $\mathcal{H}_{+}$into $\mathcal{H}_{-}$, and strongly continuous with respect to $\lambda>0$.

Our next assumption is:

(A-2) There exist unitary operators $U_{ \pm}(\lambda, \varepsilon)$ on $\mathcal{H}$ having the following properties $(\lambda, \varepsilon>0)$.

(1) For an arbitrary $g \in \mathcal{H}$,

$$
\begin{aligned}
& U_{ \pm}(\lambda, \varepsilon) R_{1}(\lambda \pm i \varepsilon) g \in D\left(H_{2}\right), \\
& U_{ \pm}^{*}(\lambda, \varepsilon) R_{2}(\lambda \pm i \varepsilon) g \in D\left(H_{1}\right),
\end{aligned}
$$

where $D\left(H_{j}\right)$ denotes the domain of $H_{j}$, and * denotes the adjoint in $\mathcal{H}$.

(2) We define

$$
\begin{aligned}
& G_{21}(\lambda \pm i \varepsilon)=\left(H_{2}-(\lambda \pm i \varepsilon)\right) U_{ \pm}(\lambda, \varepsilon) R_{1}(\lambda \pm i \varepsilon), \\
& G_{12}(\lambda \pm i \varepsilon)=\left(H_{1}-(\lambda \pm i \varepsilon)\right) U_{ \pm}^{*}(\lambda, \varepsilon) R_{2}(\lambda \pm i \varepsilon) .
\end{aligned}
$$

For every $\lambda>0, \varepsilon>0, G_{j k}(\lambda \pm i \varepsilon) \in \boldsymbol{B}\left(\widetilde{\mathcal{H}_{+}}: \mathscr{H}_{+}\right)$, and $\mathrm{s}-\lim _{\varepsilon \rightarrow 0} G_{j k}(\lambda \pm i \varepsilon)$ $\equiv G_{j k}(\lambda \pm i 0)$ exists in $\boldsymbol{B}\left(\widetilde{\mathscr{H}}_{+}: \mathscr{H}_{+}\right)$. Horeover for an arbitrary $f \in \widetilde{\mathscr{H}}_{+}, G_{j k}(\lambda \pm i 0) f$ is a strongly continuous function of $\lambda>0$ $(j, k=1,2)$.

Let an interval $(a, b)$ be fixed, and choose an arbitrary Borel set $e$ contained in $(a, b), 0<a<b<\infty$. We define 


$$
W_{j k}^{ \pm}(e) f=\int_{e} E_{j}^{\prime}(\lambda) G_{j k}(\lambda \pm i 0) f d \lambda, \quad \text { for } \quad \forall f \in \widetilde{\mathscr{H}}_{+} .
$$

By our assumption, the integrand is an $\mathscr{H}_{-}$-valued strongly continuous function of $\lambda>0$. Hence this integral is well-defined, and $W_{j k}^{ \pm}(e)$ is a bounded linear operator from $\widetilde{\mathscr{H}}_{+}$into $\mathscr{H}_{-}$. The purpose of this section is to prove the following theorem.

Theorem 1. (1) $W_{j k}^{ \pm}(e)$, defined above, is actually an operator with range in $\mathscr{H}$ which can be uniquely extended to a partial isometry on $\mathscr{H}$ with initial sct $E_{k}(e) \mathscr{H}$ and final set $E_{j}(e) \mathcal{H}$. (We use the same notation for the extended operator.)

(2) $\left(W_{j k}^{ \pm}(e)\right)^{*}=W_{k_{j}}^{ \pm}(e)$, where $*$ denotes the adjoint in $\mathcal{H}$.

(3) $W_{j k}^{ \pm}(e)$ intertwines $H_{j}$ and $H_{k}$. That is, for an arbitrary bounded Borel function $\alpha(\lambda)$ defined on the real line

$$
\alpha\left(H_{j}\right) W_{j k}^{ \pm}(e)=W_{j k}^{ \pm}(e) \alpha\left(H_{k}\right)
$$

holds. In particular, $H_{1}$, restricted to $E_{1}(e) \mathscr{H}$, and $H_{2}$, restricted to $E_{2}(e) \mathcal{H}$, are unitarily equivalent.

For the proof of this theorem, we state a lemma which is of fundamental importance.

Lemma 1.1. Let $f(\lambda), g(\lambda)$ be $\mathscr{H}_{+}$-valued locally bounded strongly measurable functions defined on $(0, \infty)$, and $e, e^{\prime}$ be Borel sets in $(a, b)$. We put

$$
\phi=\int_{e} E_{j}^{\prime}(\lambda) f(\lambda) d \lambda, \quad \phi=\int_{e^{\prime}} E_{j}^{\prime}(\lambda) g(\lambda) d \lambda .
$$

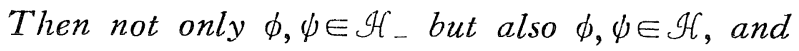

$$
(\phi, \phi)=\int_{e \cap \epsilon^{\prime}}\left(E_{j}^{\prime}(\lambda) f(\lambda), g(\lambda)\right) d \lambda
$$

holds.

Proof. First we consider the case that $f(\lambda) \equiv f, g(\lambda) \equiv g$ do not depend on $\lambda$. In this case, by the well-known Stieltjes inversion formula, we have for an arbitrary $h \in \mathscr{H}_{+}$, 


$$
\begin{aligned}
(\phi, h) & =\int_{e}\left(E_{j}^{\prime}(\lambda) f, h\right) d \lambda \\
& =\lim _{\varepsilon \downarrow 0} \int_{e}\left(\frac{1}{2 \pi i}\left[R_{j}(\lambda+i \varepsilon)-R_{j}(\lambda-i \varepsilon)\right] f, h\right) d \lambda \\
& =\left(E_{j}(e) f, h\right) .
\end{aligned}
$$

By the fact that $\mathscr{K}_{+}$is dense in $\mathscr{K}$, we have

$$
\phi=\int_{e} E_{j}^{\prime}(\lambda) f d \lambda=E_{j}(e) f \in \mathcal{H} .
$$

Similarly $\quad \phi=\int_{e^{\prime}} E_{j}^{\prime}(\lambda) g d \lambda=E_{j}\left(e^{\prime}\right) g \in \mathcal{H}, \quad$ and

$$
\begin{aligned}
(\phi, \phi) & =\left(E_{j}(e) f, E_{j}\left(e^{\prime}\right) g\right) \\
& =\left(E_{j}\left(e \cap e^{\prime}\right) f, g\right) \\
& =\int_{e \cap e^{\prime}}\left(E_{j}^{\prime}(\lambda) f, g\right) d \lambda
\end{aligned}
$$

So, the assertion of Lemma 1.1 holds for constant $f(\lambda), g(\lambda)$.

Next we consider the case that $f(\lambda), g(\lambda)$ are step functions. In this case there exist a finite number of Borel sets $e_{m}, e_{n}$ contained in $(0, \infty)$ and a finite number of $f_{m}, g_{n} \in \mathcal{H}_{+}$such that $f(\lambda)=\sum_{m} \chi_{e_{m}}(\lambda) f_{m}$, $g(\lambda)=\sum_{n} \chi_{e_{n}}(\lambda) g_{n}$, where $\chi_{e_{m}}(\lambda)$ and $\chi_{e_{n}}(\lambda)$ are the characteristic functions of $e_{m}$ and $e_{n}$, respectively. Then we have

$$
\begin{aligned}
\phi & =\int_{e} E_{j}^{\prime}(\lambda) f(\lambda) d \lambda \\
& =\sum_{m} \int_{e \cap e_{m}} E_{j}^{\prime}(\lambda) f_{m} d \lambda \\
& =\sum_{m} E_{j}\left(e \cap e_{m}\right) f_{m},
\end{aligned}
$$

and similarly $\phi=\sum_{n} E_{j}\left(e^{\prime} \cap e_{n}\right) g_{n}$. Hence $\phi, \phi \in \mathcal{H}$, and

$$
\begin{aligned}
(\phi, \phi) & =\sum_{m, n}\left(E_{j}\left(e \cap e_{m}\right) f_{m}, E_{j}\left(e^{\prime} \cap e_{n}\right) g_{n}\right) \\
& =\sum_{m, n}\left(E_{j}\left(e \cap e_{m} \cap e^{\prime} \cap e_{n}\right) f_{m}, g_{n}\right) \\
& =\sum_{m, n} \int_{e \cap e_{m} \cap e^{\prime} \cap e_{n}}\left(E_{j}^{\prime}(\lambda) f_{m}, g_{n}\right) d \lambda
\end{aligned}
$$




$$
\begin{aligned}
& =\int_{e\urcorner e^{\prime}}\left(E_{j}^{\prime}(\lambda) \sum_{m} \chi_{e_{m}}(\lambda) f_{m}, \sum_{n} \chi_{e_{n}}(\lambda) g_{n}\right) d \lambda \\
& =\int_{e \cap e^{\prime}}\left(E_{j}^{\prime}(\lambda) f(\lambda), g(\lambda)\right) d \lambda,
\end{aligned}
$$

which proves (1.5) when $f(\lambda)$ and $g(\lambda)$ are step functions.

Finally we consider the case that $f(\lambda), g(\lambda)$ are strongly measurable functions. In this case there exist sequences of step functions $\left\{f_{m}(\lambda)\right\}$, $\left\{g_{n}(\lambda)\right\}$ such that $f_{m}(\lambda) \rightarrow f(\lambda), g_{n}(\lambda) \rightarrow g(\lambda)$ in $\mathcal{H}_{+}$almost everywhere. Writing $\phi_{m}=\int_{e} E_{j}^{\prime}(\lambda) f_{m}(\lambda) d \lambda, \phi_{n}=\int_{e^{\prime}} E_{j}^{\prime}(\lambda) g_{n}(\lambda) d \lambda$, we have $\phi_{m} \rightarrow \phi$, $\psi_{n} \rightarrow \psi$ in $\mathcal{H}_{-}$. But in view of (1.5) valid for step functions we have

$$
\left(\phi_{m}-\phi_{n}, \phi_{m}-\phi_{n}\right)=\int_{e}\left(E_{j}^{\prime}(\lambda)\left(f_{m}(\lambda)-f_{n}(\lambda)\right), f_{m}(\lambda)-f_{n}(\lambda)\right) d \lambda
$$

So, there exists $\widetilde{\phi} \in \mathcal{H}$ such that $\phi_{m} \rightarrow \widetilde{\phi}$ in $\mathcal{H}$, by Lebesgue's dominated convergence theorem. But since $\phi_{m} \rightarrow \widetilde{\phi}$ in $\mathcal{H}_{-}$also, we have $\phi=\widetilde{\phi} \in \mathcal{H}$, and $\phi_{m} \rightarrow \phi$ in $\mathcal{H}$. In the same way, we see $\phi \in \mathcal{H}$, and $\phi_{n} \rightarrow \phi$ in $\mathscr{H}$. Again in view of (1.5) valid for step functions we have

$$
\left(\phi_{m}, \psi_{n}\right)=\int_{e \cap e^{\prime}}\left(E_{j}^{\prime}(\lambda) f_{m}(\lambda), g_{n}(\lambda)\right) d \lambda
$$

Letting $m, n$ tend to infinity, we see $\phi_{m} \rightarrow \phi, \psi_{n} \rightarrow \phi$ in $\mathcal{H}$ and $f_{m}(\lambda) \rightarrow f(\lambda)$, $g_{n}(\lambda) \rightarrow g(\lambda)$ in $\mathcal{H}_{+}$. Hence,

$$
(\phi, \phi)=\int_{e \cap e^{\prime}}\left(E_{j}^{\prime}(\lambda) f(\lambda), g(\lambda)\right) d \lambda
$$

which completes the proof of Lemma 1.1.

Q.E.D.

Lemma 1.2. Let $f, g \in \widetilde{\mathcal{H}}_{+}$, and $e, e^{\prime}$ be Borel sets contained in $(a, b)$. We have

$$
W_{j k}^{ \pm}(e) f, W_{j k}^{ \pm}\left(e^{\prime}\right) g \in \mathcal{H}
$$

and

$$
\begin{aligned}
\left(W_{j k}^{ \pm}(e) f, W_{j k}^{ \pm}\left(e^{\prime}\right) g\right) & =\int_{e \cap e^{\prime}}\left(E_{k}^{\prime}(\lambda) f, g\right) d \lambda \\
& =\left(E_{k}\left(e \cap e^{\prime}\right) f, g\right)
\end{aligned}
$$

holds. 
Proof. Let us define $f(\lambda)=G_{j k}(\lambda \pm i 0) f, g(\lambda)=G_{j k}(\lambda \pm i 0) g$. By the assumption (A-2), $f(\lambda)$ and $g(\lambda)$ are $\mathscr{K}_{+}$-valued strongly continuous functions of $\lambda>0$. Hence we have by (1.4) and (1.5) of Lemma 1.1, $W_{j k}^{ \pm}(e) f, W_{j k}^{ \pm}\left(e^{\prime}\right) g \in \mathcal{H}$, and

$$
\left(W_{j k}^{ \pm}(e) f, W_{j k}^{ \pm}\left(e^{\prime}\right) g\right)=\int_{e \cap e^{\prime}}\left(E_{j}^{\prime}(\lambda) G_{j k}(\lambda \pm i 0) f, G_{j k}(\lambda \pm i 0) g\right) d \lambda .
$$

Now, using the definition of $G_{j k}(\lambda \pm i \varepsilon)$ and the unitarity of $U_{ \pm}(\lambda, \varepsilon)$, we have

$$
\begin{aligned}
& \left(\frac{1}{2 \pi i}\left[R_{j}(\lambda+i \varepsilon)-R_{j}(\lambda-i \varepsilon)\right] G_{j k}(\lambda \pm i \varepsilon) f, G_{j k}(\lambda \pm i \varepsilon) g\right) \\
& \quad=\left(G_{j k}^{*}(\lambda \pm i \varepsilon) \frac{1}{2 \pi i}\left[R_{j}(\lambda+i \varepsilon)-R_{j}(\lambda-i \varepsilon)\right] G_{j k}(\lambda \pm i \varepsilon) f, g\right) \\
& \quad=\left(\frac{\varepsilon}{\pi} R_{k}(\lambda+i \varepsilon) R_{k}(\lambda-i \varepsilon) f, g\right) \\
& \quad=\left(\frac{1}{2 \pi i}\left[R_{k}(\lambda+i \varepsilon)-R_{k}(\lambda-i \varepsilon)\right] f, g\right) .
\end{aligned}
$$

Letting $\varepsilon$ tend to 0 , we have

$$
\left(E_{j}^{\prime}(\lambda) G_{j k}(\lambda \pm i 0) f, G_{j k}(\lambda \pm i 0) g\right)=\left(E_{k}^{\prime}(\lambda) f, g\right) .
$$

From this, (1.6) immediately follows.

Q.E.D.

Taking into account that $\mathcal{H}_{+}$is dense in $\mathcal{K}$, we see by Lemma 1.2 that $W_{j k}^{ \pm}(e)$ can be uniquely extended to a partial isometry on $\mathscr{H}$ with the initial set $E_{k}(e) \mathscr{H}$. We use the same notation for this extension.

Lemma 1.3. Let $e$ be a Borel set contained in $(a, b)$. We have

$$
\left(W_{j k}^{ \pm}(e)\right)^{*}=W_{k_{j}}^{ \pm}(e),
$$

where the adjoint is taken in $\mathscr{H}$.

Proof. In the proof of Lemma 1.2, we have seen

$$
\begin{aligned}
G_{j k}^{*}(\lambda \pm i \varepsilon) \frac{1}{2 \pi i}\left[R_{j}(\lambda+i \varepsilon)-R_{j}(\lambda-i \varepsilon)\right] G_{j k}(\lambda \pm i \varepsilon) \\
=\frac{1}{2 \pi i}\left[R_{k}(\lambda+i \varepsilon)-R_{k}(\lambda-i \varepsilon)\right] .
\end{aligned}
$$


Since $G_{k j}^{*}(\lambda \pm i \varepsilon) G_{j k}^{*}(\lambda \pm i \varepsilon)=I$, which follows from (A-2), multiplying both sides of the above equality by $G_{k j}^{*}(\lambda \pm i \varepsilon)$ leads to

$$
\begin{aligned}
& \frac{1}{2 \pi i}\left[R_{j}(\lambda+i \varepsilon)-R_{j}(\lambda-i \varepsilon)\right] G_{j k}(\lambda \pm i \varepsilon) \\
& =G_{k_{j}}^{*}(\lambda \pm i \varepsilon) \frac{1}{2 \pi i}\left[R_{k}(\lambda+i \varepsilon)-R_{k}(\lambda-i \varepsilon)\right] .
\end{aligned}
$$

Hence for $f, g \in \widetilde{\mathcal{H}}_{+}$, we have

$$
\begin{aligned}
(f, & \left.\frac{1}{2 \pi i}\left[R_{j}(\lambda+i \varepsilon)-R_{j}(\lambda-i \varepsilon)\right] G_{j k}(\lambda \pm i \varepsilon) g\right) \\
& =\left(f, G_{k j}^{*}(\lambda \pm i \varepsilon) \frac{1}{2 \pi i}\left[R_{k}(\lambda+i \varepsilon)-R_{k}(\lambda-i \varepsilon)\right] g\right) \\
& =\left(\frac{1}{2 \pi i}\left[R_{k}(\lambda+i \varepsilon)-R_{k}(\lambda-i \varepsilon)\right] G_{k j}(\lambda \pm i \varepsilon) f, g\right) .
\end{aligned}
$$

Letting $\varepsilon$ tend to 0 , we have

$$
\left(f, E_{j}^{\prime}(\lambda) G_{j k}(\lambda \pm i 0) g\right)=\left(E_{k}^{\prime}(\lambda) G_{k j}(\lambda \pm i 0) f, g\right) .
$$

Integrating both sides with respect to $\lambda$ on $e$ yields

$$
\left(f, W_{j k}^{ \pm}(e) g\right)=\left(W_{k_{j}}^{ \pm}(e) f, g\right),
$$

from which the assertion of the lemma readily follows.

Q.E.D.

In particular, we see by Lemma 1.3 that the final set of $W_{j k}^{ \pm}(e)$ equals the initial set of $W_{k_{j}}^{ \pm}(e)$, which is just $E_{j}(e) \mathcal{H}$.

Lemma 1.4. For an arbitrary bounded Borel function $\alpha(\lambda)$ defined on the real line, the following formula holds:

$$
\alpha\left(H_{j}\right) W_{j k}^{ \pm}(e)=W_{j k}^{ \pm}(e) \alpha\left(H_{k}\right) .
$$

Proof. Let us show the following equality

$$
E_{j}\left(e^{\prime}\right) W_{j k}^{ \pm}(e)=W_{j k}^{ \pm}(e) E_{k}\left(e^{\prime}\right),
$$

where $e^{\prime}$ is an arbitrary Borel set on the real line, and $e$ is a Borel set in $(a, b)$. It suffices to show (1.8) in the case that $e^{\prime}$ is contained in $(a, b)$, because the initial and the final sets of $W_{j k}^{ \pm}(e)$ are $E_{k}(e) \mathscr{H}$ 
and $E_{j}(e) \mathcal{H}$, respectively.

For arbitrary $f, g \in \widetilde{\mathscr{H}}_{+}$, we set $f(\lambda)=G_{j k}(\lambda \pm i 0) f, g(\lambda)=g$. By Lemma 1.1, the next formula holds for an arbitrary Borel set $e^{\prime}$ in $(a, b)$.

$$
\begin{aligned}
\left(E_{j}\left(e^{\prime}\right) W_{j k}^{ \pm}(e) f, g\right) & =\left(W_{j k}^{ \pm}(e) f, E_{j}\left(e^{\prime}\right) g\right) \\
& =\int_{e\left\lceil e^{\prime}\right.}\left(E_{j}^{\prime}(\lambda) G_{j k}(\lambda \pm i 0) f, g\right) d \lambda
\end{aligned}
$$

The right hand side of this equality is rewritten as follows.

$$
\begin{array}{rlr}
\int_{e \cap e^{\prime}} & \left(E_{j}^{\prime}(\lambda) G_{j k}(\lambda \pm i 0) f, g\right) d \lambda & \\
& =\int_{e \cap e^{\prime}}\left(f, E_{k}^{\prime}(\lambda) G_{k^{\prime} j}(\lambda \pm i 0) g\right) d \lambda & \\
& =\left(f, E_{k}\left(e^{\prime}\right) W_{k_{j}}^{ \pm}(e) g\right) & \\
& =\left(f, E_{k}\left(e^{\prime}\right)\left(W_{j k}^{ \pm}(e)\right)^{*} g\right) & \\
& =\left(W_{j k}^{ \pm}(e) E_{k}\left(e^{\prime}\right) f, g\right) . &
\end{array}
$$

Hence we have

$$
\left(E_{j}\left(e^{\prime}\right) W_{j k}^{ \pm}(e) f, g\right)=\left(W_{j k}^{ \pm}(e) E_{k}\left(e^{\prime}\right) f, g\right),
$$

which proves (1.8).

Approximating $\alpha(\lambda)$ by a sequence of step functions, in view of (1.8), we can conclude the assertion of the lemma.

Q.E.D.

Now, it is easy to see that all the assertions of Theorem 1 hold in view of Lemmas 1.2, 1.3 and 1. 4 .

Remark: The above argument is "local" in the sense that it is restricted to a bounded interval $(a, b)$. However, if we define for an arbitrary $f \in \widetilde{\mathcal{H}}_{+}$

$$
W_{j k}^{ \pm} f=\lim _{\substack{b \rightarrow \infty \\ a \rightarrow 0}} \int_{a}^{b} E_{j}^{\prime}(\lambda) G_{j k}(\lambda \pm i 0) f d \lambda,
$$

then $W_{j k}^{ \pm}$is uniquely extended to a partial isometry on $\mathcal{H}$, with the initial set $E_{k}((0, \infty)) \mathcal{H}$ and the final set $E_{j}((0, \infty)) \mathcal{H},\left(W_{j k}^{ \pm}\right) *=W_{k j}^{ \pm}$, and 
moreover $W_{j k}^{ \pm}$intertwines $H_{j}$ and $H_{k}$. Thus, we can obtain a "global" wave operator.

\section{$\S 2$. Some Remarks on the Limiting Absorption}

Consider the Schrödinger operator $H=-\Delta+V(x)$ in $L_{2}\left(\mathbb{R}^{n}\right)$, ( $\Delta$ denotes the Laplacian in $\boldsymbol{R}^{n}$ ). In this section we assume on the potential the following condition:

(C) There exists a constant $\delta(0<\delta \leq 1 / 2)$ such that $\Gamma^{\top}(x)$ is a real $C^{m}$-function and

$$
\begin{aligned}
& \Gamma^{r}(x)=O\left(|x|^{-\delta}\right), \\
& D^{k} V(x)=O\left(|x|^{-k-\delta}\right) \text { as } \quad|x| \rightarrow \infty \quad(1 \leq k \leq m),
\end{aligned}
$$

zohere $D^{k}$ denotes an arbitrary derivative of $k$-th order, and

$$
m= \begin{cases}2 / \delta+1 & (\text { if } 2 / \delta \text { is an integer }) \\ {[2 / \delta]+2} & (\text { otherwise })\end{cases}
$$

Here $[2 / \delta]$ denotes the greatest integer not exceeding $2 / \delta$.

We introduce a real $C^{\infty}$-function $\psi$ such that

$$
\psi(x)= \begin{cases}0 & (|x|<1) \\ 1 & (|x|>2)\end{cases}
$$

and decompose $V(x)$ as $V(x)=V_{1}(x)+V_{2}(x)$, where $V_{1}=\psi V, V_{2}=$ $(1-\psi) V$. Then $V_{1}$ and $V_{2}$ satisfy the following conditions:

$(\mathbf{C}-\mathbf{1})^{\prime} \quad V_{1}(x)$ is a real $C^{m}$-function such that

$$
\begin{aligned}
& D^{k} V_{1}(x)=O\left(|x|^{-k-\delta}\right) \text { as } \quad|x| \rightarrow \infty \quad(0 \leq k \leq m), \\
& V_{1}(x)=0 \quad(|x|<1) .
\end{aligned}
$$

(C-2)' $V_{2}(x)$ is a bounded real function with compact support.

Remark: Our assumption on $V(x)$ is stronger than actually needed. $V(x)$ can have certain singularities. But for the sake of simplicity, we 
continue our argument under the condition stated above.

Now, the limiting absorption method tells us a way for finding a solution of the inhomogeneous Schrödinger equation. First we list up some notations.

$L_{2, \beta}(\boldsymbol{G})$ denotes the Hilbert space of all measurable functions $f$ such that $(1+|x|)^{\beta} f(x)$ is square integrable over a domain $\boldsymbol{G} \subset \boldsymbol{R}^{n}$. The norm of $L_{2, \boldsymbol{\beta}}(\boldsymbol{G})$ is denoted by \|\|$_{\boldsymbol{\beta}, \boldsymbol{G}}$. When $\beta=0$ or $\boldsymbol{G}=\boldsymbol{R}^{n}$, we often omit the subscript.

$$
\begin{aligned}
& \tilde{x}_{j}=x_{j} / r, r=|x|, \quad(j=1, \cdots, n) . \\
& \widetilde{x}=\left(\widetilde{x}_{1}, \cdots, \widetilde{x}_{n}\right) . \\
& K_{+}=\left\{\kappa=\kappa_{1}+i \kappa_{2} \in C: \kappa_{1} \in(a, b), \kappa_{2} \in(0,1)\right\}, \\
& K_{-}=\left\{\kappa=\kappa_{1}+i \kappa_{2} \in C: \kappa_{1} \in(-b,-a), \kappa_{2} \in(0,1)\right\},
\end{aligned}
$$

where $a, b$ are arbitrary positive constants such that $a<b$.

$$
\begin{aligned}
& \mathscr{D}_{j}=\frac{\partial}{\partial x_{j}}+\frac{n-1}{2 r} \widetilde{x}_{j}-i \kappa \widetilde{x}_{j}\left(\kappa \in K_{ \pm}, 1 \leq j \leq n\right) . \\
& \mathscr{D}_{r}=\frac{\partial}{\partial r}+\frac{n-1}{2 r}-i \kappa=\sum_{j} \widetilde{x}_{j} \mathscr{D}_{j} . \\
& \mathscr{D}=\left(\mathscr{D}_{1}, \mathscr{D}_{2}, \cdots, \mathscr{D}_{n}\right) . \\
& \widetilde{\operatorname{grad}}=\operatorname{grad}-\widetilde{x} \frac{\partial}{\partial r} .
\end{aligned}
$$

$H_{\text {loc }}^{2}$ is all $L_{2, \text { loc }}$ functions with $L_{2, \text { loc }}$ distribution derivatives up to the second order, inclusive.

Under our assumption on $V(x), H=-\Delta+V(x)$ is, when restricted to $C_{0}^{\infty}\left(\boldsymbol{R}^{n}\right)$, essentially self-adjoint. We use the same notation $H$ for its unique self-adjoint extension. Further, we adopt the following notations.

$$
R\left(\kappa^{2}\right)=\left(H-\kappa^{2}\right)^{-1} \quad\left(\kappa \in K_{ \pm}\right) .
$$

$E(\lambda)$ is the resolution of the identity for $H$.

$$
u(\kappa: f)=R\left(\kappa^{2}\right) f .
$$

$\varepsilon_{0}$ is a positive constant such that $0<\varepsilon_{0} \leq \delta / 2$. 


$$
\begin{aligned}
& E_{\rho}=\left\{x \in \mathbb{R}^{n}:|x| \geq \rho\right\} \quad(\rho>0) . \\
& B_{\rho}=\left\{x \in \boldsymbol{R}^{n}:|x| \leq \rho\right\} \quad(\rho>0) . \\
& B_{\rho, \sigma}=\left\{x \in \boldsymbol{R}^{n}: \rho<|x|<\sigma\right\} \quad(0<\rho<\sigma) .
\end{aligned}
$$

The following theorem is due to Ikebe-Saito [7].

Theorem 2. (a) The following a-priori estimates hold:

$$
\begin{aligned}
& \|u(\kappa: f)\|_{-\left(1+\varepsilon_{0}\right) / 2} \leq C\|f\|_{\left(1+\varepsilon_{0}\right) / 2}, \\
& \|\mathscr{D} u(\kappa: f)\|_{-\left(1-\varepsilon_{0}\right) / 2, E_{1}} \leq C\|f\|_{\left(1+\varepsilon_{0}\right) / 2},
\end{aligned}
$$

where $C$ is a constant which does not depend on $f \in L_{2,\left(1+\varepsilon_{0}\right) / 2}$ and $\kappa \in K_{ \pm}$.

(b) (Limiting absorption method) $u(\kappa: f)$ is continuous in $L_{2,-\left(1+\varepsilon_{0}\right) / 2}$ with respect to $\kappa \in K_{ \pm}$and $f \in L_{2,\left(1+\varepsilon_{0}\right) / 2}$, and for any $\lambda>0$ $(a<\lambda<b)$, the limit

$$
u( \pm \sqrt{\lambda}+i 0: f)=\lim _{\varepsilon \rightarrow 0} u( \pm \sqrt{\lambda}+i \varepsilon: f)=R(\lambda \pm i 0) f
$$

exists in $L_{2,-\left(1+\varepsilon_{0}\right) / 2}$, and the inequalities stated in (a) are satisfied with $u=u( \pm \sqrt{\lambda}+i 0: f)$.

(c) For any pair $(\kappa, f) \in \bar{K}_{ \pm} \times L_{2,\left(1+\varepsilon_{0}\right) / 2}$, where $\bar{K}_{ \pm}$is the closure of $K_{ \pm}$in $\mathbb{C}$, there exists a unique solution $u=u(\kappa: f) \in L_{2,-\left(1+\varepsilon_{0}\right) / 2} \cap H_{\mathrm{loc}}^{2}$ of

$$
\left(H-\kappa^{2}\right) u=f, \quad\|\mathscr{D} u\|_{-\left(1-\varepsilon_{0}\right) / 2, E_{1}}<\infty
$$

The mapping

$$
\bar{K}_{ \pm} \times L_{2,\left(1+\varepsilon_{0}\right) / 2} \ni(\kappa, f) ! \rightarrow u(\kappa: f) \in L_{2,\left(1+\varepsilon_{0}\right) / 2}
$$

is continuous on $\bar{K}_{ \pm} \times L_{2,\left(1+\varepsilon_{0}\right) / 2}$.

(d) For $f, g \in L_{2,\left(1+\varepsilon_{0}\right) / 2}$ and any Borel set $e \subset(0, \infty)$ we have

$$
(E(e) f, g)=\frac{1}{2 \pi i} \int_{e}(R(\lambda+i 0) f-R(\lambda-i 0) f, g) d \lambda .
$$

The part of $H$ in $E((0, \infty)) L_{2}\left(\boldsymbol{R}^{n}\right)$ is absolutely continuous.

Remark: We say that a function $u(x)$ satisfies the radiation condition if it satisfies the following inequality 


$$
\|\mathscr{D} u\|_{-\left(1-\varepsilon_{0}\right) / 2, E_{1}}<\infty \text {. }
$$

Now, for a real constant $\kappa_{1}\left(a<\kappa_{1}<b,-b<\kappa_{1}<-a\right)$, we consider the following non-linear equation

$$
2 \kappa_{1} \frac{\partial X}{\partial r}=V_{1}(x)+|\nabla X|^{2}
$$

where $\nabla$ denotes the gradient on $\boldsymbol{R}^{n}$. A successive approximation scheme for the above equation is

$$
\begin{aligned}
& X^{(0)}\left(x, \kappa_{1}\right)=0, \\
& \begin{aligned}
& 2 \kappa_{1} X^{(j)}\left(x, \kappa_{1}\right)= \int_{0}^{r}\left(V_{1}(s \widetilde{x})+\left|\left(\nabla X^{(j-1)}\right)\left(s \widetilde{x}, \kappa_{1}\right)\right|^{2}\right) d s \\
& \quad+\phi_{j}\left(\widetilde{x}, \kappa_{1}\right) \rho(x), \\
& r=|x|, j=1,2, \cdots,
\end{aligned}
\end{aligned}
$$

where the function $\phi_{j}\left(\widetilde{x}, \kappa_{1}\right)$ is defined by

$$
\phi_{j}\left(\widetilde{x}, \kappa_{1}\right)=\left\{\begin{array}{l}
0 \quad \text { if } \quad j \delta<1 \\
\phi_{j-1}\left(\widetilde{x}, \kappa_{1}\right)-\int_{0}^{\infty} A_{j-1}\left(s \widetilde{x}, \kappa_{1}\right) d s \text { if } j \delta>1,
\end{array}\right.
$$

where $A_{j}\left(x, \kappa_{1}\right)=\left|\left(\nabla X^{(j)}\right)\left(x, \kappa_{1}\right)\right|^{2}-\left|\left(\nabla X^{(j-1)}\right)\left(x, \kappa_{1}\right)\right|^{2}$, and $\rho(x)$ is a real $C^{\infty}$-function such that

$$
\rho(x)= \begin{cases}0 & |x|<1 \\ 1 & |x|>2\end{cases}
$$

Here we should remark that without loss of generality we can assume $1 / \delta$ is not an integer. The following lemma concerning the $j$-th approximation can be proved by induction on $j$.

Lemma 2. 1. $X^{(j)}\left(x, \kappa_{1}\right)=0$ if $|x|<1$,

$$
\begin{aligned}
& \left|D^{k} X^{(j)}\left(x, \kappa_{1}\right)\right| \leq C(1+|x|)^{1-k-\delta} \quad(0 \leq k \leq m-j+1), \\
& \left.\left|2 \kappa_{1} \frac{\partial}{\partial r} X^{(j)}\left(X, \kappa_{1}\right)-V_{1}(x)-\right|\left(\nabla X^{(j)}\right)\left(x, \kappa_{1}\right)\right|^{2} \mid \\
& \quad \leq C(1+|x|)^{-(j+1) \delta}
\end{aligned}
$$

where the constant $C$ does not depend on $\kappa_{1}\left(a<\kappa_{1}<b,-b<\kappa_{1}<-a\right)$. 
We choose the smallest positive integer $j$ such that $(j+1) \delta \geq 2$ and difine $X\left(x, \kappa_{1}\right)=X^{(j)}\left(x, \kappa_{1}\right)$. Note that $X\left(x, \kappa_{1}\right)$ is a real $C^{3}$-function of $x$ and $\kappa_{1}$ having the following properties:

$$
\begin{aligned}
& X\left(x, \kappa_{1}\right)=0 \quad \text { if } \quad|x|<1, \\
& \left|D^{k} X\left(x, \kappa_{1}\right)\right| \leq C(1+|x|)^{-k+1-\delta} \quad(1 \leq k \leq 3), \\
& \left.\left|2 \kappa_{1} \frac{\partial}{\partial r} X\left(x, \kappa_{1}\right)-V_{1}(x)-\right|(\nabla X)\left(x, \kappa_{1}\right)\right|^{2} \mid \leq C(1+|x|)^{-2},
\end{aligned}
$$

where the constant $C$ does not depend on $\kappa_{1}\left(a<\kappa_{1}<b,-b<\kappa_{1}<-a\right)$.

We put $v=v(\kappa: f)=e^{i X\left(x, \kappa_{1}\right)} u(\kappa: f)$, where $f \in L_{2,\left(3-\varepsilon_{0}\right) / 2}, \kappa=\kappa_{1}+i \kappa_{2}$, $\kappa \in \bar{K}_{ \pm}$. Then we can prove the following a-priori estimate concerning the radiation condition.

Lemma 2. 2. $\|\mathscr{D} v\|_{\left(1-\varepsilon_{0}\right) / 2, E_{1}} \leq C\|f\|_{\left(3-\varepsilon_{0}\right) / 2}$, where the constant $C$ depends neither on $f \in L_{2,\left(3-\varepsilon_{0}\right) / 2}$ nor on $\kappa \in \bar{K}_{ \pm}$.

The proof of this lemma is somewhat long and complicated, so we shall prove it in the Appendix.

Lemma 2.3. $\|\mathscr{D} v\|_{\left(1-3 \varepsilon_{0}\right) / 2, E_{\rho}} \leq C \rho^{-\varepsilon_{0}}\|f\|_{\left(3-\varepsilon_{0}\right) / 2} \quad(\forall \rho>1)$.

Proof. This lemma follows easily from the following inequalities:

$$
\begin{aligned}
\|\mathscr{D} v\|_{\left(1-3 \varepsilon_{0}\right) / 2, E_{\rho}}^{2} & =\int_{|x|>\rho}(1+|x|)^{1-\varepsilon_{0}-2 \varepsilon_{0}}|\mathscr{D} v|^{2} d x \\
& \leq(1+\rho)^{-2 \varepsilon_{0}} \int_{|x|>\rho}(1+|x|)^{1-\varepsilon_{0}}|\mathscr{D} v|^{2} d x \\
& \leq(1+\rho)^{-2 \varepsilon_{0}} C\|f\|^{2}{ }_{\left(3-\varepsilon_{0}\right) / 2}
\end{aligned}
$$

(by Lemma 2.2). Q.E.D.

Lemma 2. 4. $\kappa_{2}\|v\|_{\left(1-\varepsilon_{0}\right) / 2} \leq C\|f\|_{\left(3-\varepsilon_{0}\right) / 2} \quad\left(\kappa \in \bar{K}_{ \pm}\right)$.

Proof. In Ikebe-Saitō ([7], Lemma 2.3), the following inequality is proved:

$$
\kappa_{2}\|u\|_{\left(1-\varepsilon_{0}\right) / 2} \leq C\left(\|u\|_{-\left(1+\varepsilon_{0}\right) / 2}+\|\mathscr{D} u\|_{-\left(1-\varepsilon_{0}\right) / 2, E_{1}}+\|f\|_{\left(1+\varepsilon_{0}\right) / 2}\right) .
$$


Taking into account that $X\left(x, \kappa_{1}\right)$ is a real function, we have for an arbitrary real constant $\beta$

$$
\|v\|_{\beta}=\left\|e^{i x} u\right\|_{\beta}=\|u\|_{\beta} .
$$

In view of Theorem 2 (a), the right hand side of (2.2) is estimated from above as follows:

$$
\text { (the right hand side of } \begin{aligned}
(2.2)) & \leq C\|f\|_{\left(1+\varepsilon_{0}\right) / 2} \\
& \leq C\|f\|_{\left(3-\varepsilon_{0}\right) / 2} .
\end{aligned}
$$

By (2.2), (2.3) and (2.4), the assertion of the lemma readily follows.

Q.E.D.

Lemma 2. 5. $\kappa_{2}\|v\|_{\left(1-3 \varepsilon_{0}\right) / 2, E_{\rho}} \leq C \rho^{-\varepsilon_{0}}\|f\|_{\left(3-\varepsilon_{0}\right) / 2} \quad(\forall \rho>1)$.

This lemma is proved in the same way as in Lemma 2.3.

Remark: It is easy to see that in Lemmas 2.3, 2.4 and 2.5, the constant $C$ does not depend on $\kappa \in \bar{K}_{ \pm}$.

Lemma 2.6. $\operatorname{s}_{\kappa \rightarrow \pm \sqrt{\bar{\lambda}}+i 0} \kappa_{2} v(\kappa: f)=0$ in $L_{2,\left(1-3 \varepsilon_{0}\right) / 2}$.

Proof. By Lemma 2.5, for an arbitrary $\varepsilon>0$, there exists a constant $r_{0}>0$ such that $\kappa_{2}\|v\|_{\left(1-3 \varepsilon_{0}\right) / 2, E_{r_{0}}}<\varepsilon$, where $r_{0}$ is independent of $\kappa \in \bar{K}_{ \pm}$.

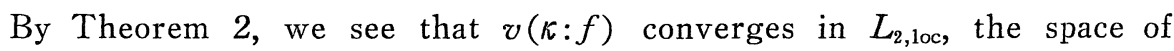
locally $L_{2}$ functions, when $\kappa$ tends to $\pm \sqrt{\lambda}+i 0$. Hence $\|v(\kappa: f)\|_{\left(1-3 \varepsilon_{0}\right) / 2, B_{0}}$ is uniformly bounded in $\kappa \in \bar{K}_{ \pm}$. So, letting $\kappa_{2}$ be sufficiently small, we have $\kappa_{2}\|v(\kappa: f)\|_{\left(1-3 \varepsilon_{0}\right) / 2, B_{r_{0}}}<\varepsilon$. These facts yield the lemma. Q.E.D.

Lemma 2. 7. When $\kappa=\kappa_{1}+i \kappa_{2}\left(\in K_{ \pm}\right)$tends to $\pm \sqrt{ } \lambda+i 0$, $\mathscr{D} v(\kappa: f)$ tends to $\left(\operatorname{grad}+\frac{n-1}{2 r} \widetilde{x} \mp i \sqrt{\lambda} \widetilde{x}\right) v( \pm \sqrt{ } \lambda+i 0: f)$ in $L_{2,\left(1-3 \varepsilon_{0}\right) / 2, E_{1}}$.

Proof. First we note that $u(\kappa: f)$ tends to $u( \pm \sqrt{\lambda}+i 0: f)$ in $H_{\mathrm{loc}}^{2}$, which follows from Theorem 2 and the following well-known elliptic estimate: 
(2.5) $\sum_{|\alpha| \leq 2} \int_{|x| \leq \rho}\left|D^{\alpha} u\right|^{2} d x \leq C(\rho, R)\left(\int_{|x| \leq R}|(H-z) u|^{2} d x+\int_{|x| \leq R}|u|^{2} d x\right)$, where $z \in \mathbb{C}, \rho$ and $R$ are arbitrary positive constants such that $\rho<R, u(x)$ is an arbitrary $H_{\text {loc }}^{2}$ function and $\alpha$ is a multi-index. From this fact we can conclude that $v(\kappa: f)$ tends to $v( \pm \sqrt{\lambda}+i 0: f)$ in $H_{\text {loc }}^{2}$ By Lemma 2.3 , for an arbitrary $\varepsilon>0$, there exists a constant $r_{0}>0$ such that $\left\|\mathscr{D}_{v}\right\|_{\left(1-3 \varepsilon_{0}\right) / 2, E_{r_{0}}}<\varepsilon$, where $r_{0}>1$ and is independent of $\kappa \in \bar{K}_{ \pm}$. If we take $\kappa$ sufficiently close to $\pm \sqrt{\lambda}$, we have

$$
\left\|\mathscr{D} v(\kappa: f)-\left(\operatorname{grad}+\frac{n-1}{2 r} \tilde{x} \mp i \sqrt{ } \lambda \tilde{x}\right) v( \pm \sqrt{\lambda}+i 0: f)\right\|_{\left(1-3 \varepsilon_{0}\right) / 2, B_{1}, r_{0}}<\varepsilon,
$$

which follows from the previous fact that $v(\kappa: f)$ tends to $v( \pm \sqrt{ } \lambda+i 0: f)$ in $H_{\text {loc. }}^{2}$ These two facts yield the lemma.

Q.E.D.

Lemma 2. 8. For an arbitrary $f \in L_{2,\left(3-\varepsilon_{0}\right) / 2}$, the following facts hold.

(1) The following inequalities hold:

$$
\left\|\left(\frac{\partial}{\partial r}+\frac{n-1}{2 r} \mp i \sqrt{\lambda}\right) v(\sqrt{\lambda \pm i \varepsilon}: f)\right\|_{\left(1-\varepsilon_{0}\right) / 2, E_{1}} \leq C\|f\|_{\left(3-\varepsilon_{0}\right) / 2},
$$

$$
\|\widetilde{\operatorname{grad}} v(\sqrt{\lambda \pm i \varepsilon}: f)\|_{\left(1-\varepsilon_{0}\right) / 2, E_{1}} \leq C\|f\|_{\left(3-\varepsilon_{0}\right) / 2},
$$

where the constant $C$ does not depend on $\lambda$ and $\varepsilon$ such that $a<\lambda<b$, $0<\varepsilon<1, \operatorname{Im} \sqrt{ } \geq 0$ (Im=imaginary part).

(2) The following two strong limits exist in $L_{2,\left(1-3 \varepsilon_{0}\right) / 2, E_{1}}$ :

$$
\begin{aligned}
& \mathrm{s}-\lim _{\varepsilon \rightarrow 0}\left(\frac{\partial}{\partial r}+\frac{n-1}{2 r} \mp i \sqrt{\lambda}\right) v(\sqrt{\lambda \pm i \bar{\varepsilon}: f)} \\
& =\left(\frac{\partial}{\partial r}+\frac{n-1}{2 r} \mp i \sqrt{ } \lambda\right) v( \pm \sqrt{\lambda}+i 0: f)
\end{aligned}
$$

(2.9) $\quad \operatorname{s-lim} \underset{\operatorname{grad}}{ } v(\sqrt{\lambda \pm i \varepsilon}: f)=\widetilde{\operatorname{grad}} v( \pm \sqrt{\lambda}+i 0: f)$.

(3) $\left(\frac{\partial}{\partial r}+\frac{n-1}{2 r} \mp i \sqrt{\lambda}\right) v( \pm \sqrt{\lambda}+i 0: f)$ and $\underset{\operatorname{grad}}{\sim}( \pm \sqrt{\lambda}+i 0: f)$ are strongly continuous for $\lambda>0$ in $L_{2,\left(1-3 \varepsilon_{0}\right) / 2, E_{1}}$.

Proof. Let us first show the assertion (1). We have 


$$
\begin{gathered}
\left(\frac{\partial}{\partial r}+\frac{n-1}{2 r} \mp i \sqrt{\lambda}\right) v(\sqrt{\lambda \pm i \varepsilon}: f) \\
=\left(\frac{\partial}{\partial r}+\frac{n-1}{2 r}-i \sqrt{\lambda \pm i \varepsilon}\right) v(\sqrt{\lambda \pm i \varepsilon}: f) \\
\mp \frac{\varepsilon}{\sqrt{\lambda \pm i \varepsilon} \pm \sqrt{\lambda}} v(\sqrt{\lambda \pm i \varepsilon}: f) .
\end{gathered}
$$

Then we have by Lemma 2.2 and Lemma 2.4 ,

$$
\begin{aligned}
& \left\|\left(\frac{\partial}{\partial r}+\frac{n-1}{2 r} \mp i \sqrt{\lambda}\right) v(\sqrt{\lambda \pm i \varepsilon}: f)\right\|_{\left(1-\varepsilon_{0}\right) / 2, E_{1}} \\
& \quad \leq\left\|\left(\frac{\partial}{\partial r}+\frac{n-1}{2 r}-i \sqrt{\lambda} \pm i \varepsilon\right) v(\sqrt{\lambda} \pm \bar{\varepsilon} \varepsilon: f)\right\|_{\left(1-\varepsilon_{0}\right) / 2, E_{1}} \\
& \quad+\quad \mid \sqrt{\lambda \pm i \varepsilon \pm \sqrt{ } \bar{\lambda} \mid}\|v(\sqrt{\lambda \pm i \varepsilon}: f)\|_{\left(1-\varepsilon_{0}\right) / 2, E_{1}} \\
& \quad \leq C\|f\|_{\left(3-\varepsilon_{0}\right) / 2}
\end{aligned}
$$

which proves (2.6). Similarly by Lemma 2.2 we have (2.7).

Next we show the assertion (2). By Lemma 2.7, the first term of the right hand side of (2.10) tends to $\left(\frac{\partial}{\partial r}+\frac{n-1}{2 r} \mp i \sqrt{\lambda}\right) v( \pm \sqrt{\lambda}+i 0: f)$ in $L_{2,\left(1-3 \varepsilon_{0}\right) / 2, E_{1}}$, and by Lemma 2.6, the second term converges to 0 in $L_{2,\left(1-3 \varepsilon_{0}\right) / 2}$ as $\varepsilon \rightarrow 0$. This proves (2.8), (2.9) is proved similarly.

To prove (3), we must first note that the mapping $\lambda \mid \rightarrow u( \pm \sqrt{\lambda}+i 0: f)$ is continuous in $H_{\text {loc }}^{2}$, which follows from (c) of Theorem 2 and the elliptic estimate (2.5). From this we can conclude that mapping $\lambda l \rightarrow v( \pm \sqrt{\lambda}+i 0: f)$ is continuous in $H_{\text {loc }}^{2}$. Now, let there be a sequence $\lambda_{m}>0(m=1,2, \cdots)$ such that $\lambda_{m} \rightarrow \lambda_{0}(>0)$ as $m \rightarrow \infty$. By Lemma 2.3, for an arbitrary $\varepsilon>0$, there exists a constant $r_{0}>0$ independent of $\lambda_{m}$ $(m=0,1,2, \cdots)$ such that $\left\|\left(\frac{\partial}{\partial r}+\frac{n-1}{2 r} \mp i \sqrt{\lambda_{m}}\right) v\left( \pm \sqrt{\lambda_{m}}+i 0: f\right)\right\|_{\left(1-8 \varepsilon_{0}\right) / 2, E_{r_{0}}}$ $<\varepsilon$. By the strong continuity of $v(\kappa: f)$ in $H_{1 \text { loc }}^{2}$, we have for sufficiently large $m$

$$
\begin{aligned}
& \|\left(\frac{\partial}{\partial r}+\frac{n-1}{2 r} \mp i \sqrt{\lambda_{m}}\right) v\left( \pm \sqrt{\lambda_{m}}+i 0: f\right) \\
& \quad-\left(\frac{\partial}{\partial r}+\frac{n-1}{2 r} \mp i \sqrt{\lambda_{0}}\right) v\left( \pm \sqrt{\lambda_{0}}+i 0: f\right) \|_{\left(1-3 \varepsilon_{0}\right) / 2, B_{1}, r_{0}}<\varepsilon .
\end{aligned}
$$


These two facts prove the strong continuity of $\left(\frac{\partial}{\partial r}+\frac{n-1}{2 r} \mp i \sqrt{\lambda}\right)$ $\times v( \pm \sqrt{\lambda}+i 0: f)$ in $L_{2,\left(1-3 \varepsilon_{0}\right) / 2, E_{1}}$. The strong continuity of $\widetilde{\operatorname{grad}} v( \pm \sqrt{\lambda}$ $+i 0: f)$ is proved similarly.

Q.E.D.

\section{$\S 3$. Existence and Unitarity of the Stationary Wave Operator}

Let $H_{1}=-\Delta, H_{2}=-J+V(x)$, where $V(x)$ satisfies the condition (C) stated in $\S 2$. Then we can prove the following theorem.

Theorem 3. When wc take $U_{ \pm}(\lambda, \varepsilon)$ as the operator of multiplication by the function $\exp (-i \mathrm{X}(x, \operatorname{Re} \sqrt{\lambda \pm i \varepsilon}))$, where $X$ is the function which has been defined in $\S 2$ and $\mathrm{Re}$ means the real part, assumptions (A-1) and (A-2) of $\S 1$ are satisfied. Hence there exist stationary wave operators $\mathrm{I}_{j^{k}}^{ \pm}(\mathrm{c})$ having the following propertics:

(1) $W_{\frac{t}{j k}(c)}$ is a partial isometry with the initial set $E_{k}(e) L_{2}\left(\boldsymbol{R}^{n}\right)$ and the final set $E_{j}(e) L_{2}\left(\boldsymbol{R}^{n}\right)$.

(2) $\left(W_{\frac{ \pm}{j k}}^{ \pm}(e)\right)^{*}=W_{\frac{1}{k j}}^{\stackrel{\perp}{c})}$, where the adjoint is taken in $L_{2}\left(\mathbb{R}^{n}\right)$.

(3) $H_{j} W_{j k}^{ \pm}(e) \supseteq W_{j k}^{ \pm}(e) H_{k}(j, k=1,2)$,

where $e$ is a Borel set in $(a, b) \quad(0<a<b<\infty)$.

Proof. Let $\mathscr{H}=L_{2}\left(\boldsymbol{R}^{n}\right), \quad \mathcal{H}_{ \pm}=L_{2, \pm\left(1+\varepsilon_{0}\right) / 2}, \quad \widetilde{\mathscr{H}}_{+}=L_{2,\left(3-\varepsilon_{0}\right) / 2} \quad$ in the notation of $\S 1$. The assumption $(\mathrm{A}-1)$ is guaranteed by Theorem 2 . To see that $(\mathrm{A}-2)$ is fulfilled, we rewrite $G_{j k}(\lambda \pm i \varepsilon)$. Let us calculate the commutator $\left[-\Delta, e^{-i X}\right]$ as follows:

$$
\begin{aligned}
{\left[-\Delta, e^{-i X}\right]=} & -\left(\Delta e^{-i X}\right)-2\left(\nabla e^{-i X}\right) \cdot \nabla \\
= & i e^{-i X}\left(\frac{\partial^{2} X}{\partial r^{2}}+\frac{\Lambda X}{r^{2}}\right)+e^{-i X}\left(|\nabla X|^{2}-2(\operatorname{Re} \sqrt{\lambda \pm i \varepsilon}) \frac{\partial X}{\partial r}\right) \\
& +2 i e^{-i X} \frac{\partial X}{\partial r}\left(\frac{\partial}{\partial r}+\frac{n-1}{2 r} \mp i \sqrt{\lambda}\right) \\
& +2 i e^{-i x} \underset{\mathrm{grad} X}{\widetilde{\mathrm{grad}}} \\
& +2\left(\operatorname{Re} \frac{ \pm i \varepsilon}{\sqrt{ } \lambda \pm i \varepsilon \pm \sqrt{ } \lambda}\right) e^{i X} \frac{\partial X}{\partial r},
\end{aligned}
$$

where $\Lambda$ denotes the Laplace-Beltrami operator on the unit sphere. 
Then we have for $f \in L_{2}\left(\boldsymbol{R}^{n}\right)$,

$$
\begin{aligned}
G_{21}(\lambda \pm i \varepsilon) f= & \left(H_{2}-(\lambda \pm i \varepsilon)\right) U_{ \pm}(\lambda, \varepsilon) R_{1}(\lambda \pm i \varepsilon) f \\
= & U_{ \pm}(\lambda, \varepsilon)\{-\Delta-(\lambda \pm i \varepsilon)\} R_{1}(\lambda \pm i \varepsilon) f \\
& +\left\{\left[-\Delta, e^{-i X}\right]+V(x) e^{-i X}\right\} R_{1}(\lambda \pm i \varepsilon) f \\
= & U_{ \pm}(\lambda, \varepsilon) f \\
& +i U_{ \pm}(\lambda, \varepsilon)\left(\frac{\partial^{2} X}{\partial r^{2}}+\frac{A X}{r^{2}}\right) R_{1}(\lambda \pm i \varepsilon) f \\
& +U_{ \pm}(\lambda, \varepsilon)\left\{|\nabla X|^{2}+V(x)-2(\operatorname{Re} \sqrt{\lambda \pm i \varepsilon}) \frac{\partial X}{\partial r}\right\} \\
& \times R_{1}(\lambda \pm i \varepsilon) f \\
& +2 i U_{ \pm}(\lambda, \varepsilon) \frac{\partial X}{\partial r}\left(\frac{\partial}{\partial r}+\frac{n-1}{2 r} \mp i \sqrt{\lambda}\right) R_{1}(\lambda \pm i \varepsilon) f \\
& +2 i U_{ \pm}(\lambda, \varepsilon) \stackrel{\operatorname{grad} X \cdot \frac{\operatorname{grad} R_{1}(\lambda \pm i \varepsilon) f}{}}{+}+2 U_{ \pm}(\lambda, \varepsilon)\left(\operatorname{Re} \frac{ \pm i \varepsilon}{\sqrt{\lambda \pm i \varepsilon} \pm \sqrt{\lambda}}\right) \frac{\partial X}{\partial r} R_{1}(\lambda \pm i \varepsilon) f .
\end{aligned}
$$

The calculation of $G_{12}(\lambda \pm i \varepsilon) f$ can be done in a similar way after computing the commutator $\left[-\Delta, e^{i X}\right]$. But in this case we must further compute $\left[e^{i x}, \nabla\right]$. Thus we get the following expression for $G_{12}(\lambda \pm i \varepsilon) f$ :

$$
\begin{aligned}
& G_{12}(\lambda \pm i \varepsilon) f=\left(H_{1}-(\lambda \pm i \varepsilon)\right) U_{ \pm}^{*}(\lambda, \varepsilon) R_{2}(\lambda \pm i \varepsilon) f \\
& =U_{ \pm}^{*}(\lambda, \varepsilon) f \\
& -i\left(\frac{\partial^{2} X}{\partial r^{2}}+\frac{\Lambda X}{r^{2}}\right) U_{ \pm}^{*}(\lambda, \varepsilon) R_{2}(\lambda \pm i \varepsilon) f \\
& -U_{ \pm}^{*}(\lambda, \varepsilon)\left\{|\nabla X|^{2}+V(x)-2(\operatorname{Re} \sqrt{\lambda \pm i \varepsilon}) \frac{\partial X}{\partial r}\right\} \\
& \times R_{2}(\lambda \pm i \varepsilon) f \\
& -2 i \frac{\partial X}{\partial r}\left(\frac{\partial}{\partial r}+\frac{n-1}{2 r} \mp i \sqrt{\lambda}\right)\left(U_{ \pm}^{*}(\lambda, \varepsilon) R_{2}(\lambda \pm i \varepsilon) f\right) \\
& -2 i \stackrel{\operatorname{grad}}{ } X \cdot \widetilde{\operatorname{grad}}\left(U_{ \pm}^{*}(\lambda, \varepsilon) R_{2}(\lambda \pm i \varepsilon) f\right) \\
& -2 U_{ \pm}^{*}(\lambda, \varepsilon)\left(\operatorname{Re} \frac{ \pm i \varepsilon}{\sqrt{\lambda \pm i \varepsilon} \pm \sqrt{\lambda}}\right) \frac{\partial X}{\partial r} R_{2}(\lambda \pm i \varepsilon) f .
\end{aligned}
$$


Now, let us show the following two assertions:

(3.4) There exists a constant $C$ which does not depend on $\lambda, \varepsilon$ $(0<a<\lambda<b, 0<\varepsilon<1)$ such that for an arbitrary $f \in L_{2,\left(3-\varepsilon_{0}\right) / 2}$,

$$
\left\|G_{j k}(\lambda \pm i \varepsilon) f\right\|_{\left(1+\varepsilon_{0}\right) / 2} \leq C\|f\|_{\left(3-\varepsilon_{0}\right) / 2}
$$

holds.

(3.5) For $f \in L_{2,\left(3-\varepsilon_{0}\right) / 2}$, there exists a strong limit $\mathrm{s}-\lim _{\varepsilon \rightarrow 0} G_{j k}(\lambda \pm i \varepsilon) f$

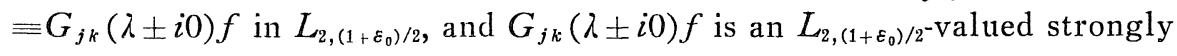
continuous function of $\lambda>0$.

First we consider $G_{12}(\lambda \pm i \varepsilon) f$. Let us first note that $e^{i X\left(x, \kappa_{1}\right)}$ is a continuous function of $\kappa_{1}$ with its derivatives. The first term of the right hand side of (3.3) is easily seen to satisfy (3.4) and (3.5), where $G_{j k}(\lambda \pm i \varepsilon) f$ is replaced with $U_{ \pm}^{*}(\lambda, \varepsilon) f$, for $U_{ \pm}^{*}(\lambda, \varepsilon)$ is just an operator of multiplication by a function with the absolute value one. Hence we have

$$
\left\|U_{ \pm}^{*}(\lambda, \hat{\varepsilon}) f\right\|_{\left(1+\varepsilon_{0}\right) / 2}=\|f\|_{\left(1+\varepsilon_{0}\right) / 2} \leq\|f\|_{\left(3-\varepsilon_{0}\right) / 2} .
$$

By Lebesgue's convergence theorem, $U_{ \pm}^{*}(\lambda, \varepsilon) f \rightarrow U_{ \pm}^{*}(\lambda, 0) f$ in $L_{2,\left(1+\varepsilon_{0}\right) / 2}$ as $\varepsilon \rightarrow 0$, and $U_{ \pm}^{*}(\lambda, 0) f$ is strongly continuous for $\lambda>0$ in $L_{2,\left(1+\varepsilon_{0}\right) / 2}$.

In veiw of Theorem $2, R_{2}(\lambda \pm i \varepsilon)$ is a bounded operator from $L_{2,\left(3-\varepsilon_{0}\right) / 2}$ into $L_{2,-\left(1+\varepsilon_{0}\right) / 2}$, and by $(2.1)$ of $\S 2$ we have

$$
\begin{aligned}
& \left|\frac{\partial^{2} X}{\partial r^{2}}+\frac{\Lambda X}{r^{2}}\right| \leq C(1+|x|)^{-1-\delta} \quad\left(\delta \geq 2 \varepsilon_{0}\right), \\
& \left.|| \nabla X\right|^{2}+V(x)-2(\operatorname{Re} \sqrt{\lambda \pm i \varepsilon}) \frac{\partial X}{\partial r} \mid \leq C(1+|x|)^{-2},
\end{aligned}
$$

where the constant $C$ is independent of $\lambda, \varepsilon(a<\lambda<b, 0<\varepsilon<1)$. Hence we have the following inequalities for the second and the third terms.

$$
\begin{gathered}
\left\|\left(\frac{\partial^{2} X}{\partial r^{2}}+\frac{\Lambda X}{r^{2}}\right) U_{ \pm}^{*}(\lambda, \varepsilon) R_{2}(\lambda \pm i \varepsilon) f\right\|_{\left(1+\varepsilon_{0}\right) / 2} \leq C\|f\|_{\left(3-\varepsilon_{0}\right) / 2} \\
\left\|U_{ \pm}^{*}(\lambda, \varepsilon)\left\{|\nabla X|^{2}+V(x)-2(\operatorname{Re} \sqrt{ } \lambda \pm i \bar{\varepsilon}) \frac{\partial X}{\partial r}\right\} R_{2}(\lambda \pm i \varepsilon) f\right\|_{\left(1+\varepsilon_{0}\right) / 2} \\
\leq C\|f\|_{\left(3-\varepsilon_{0}\right) / 2},
\end{gathered}
$$

where the constant $C$ is independent of $\lambda, \varepsilon(a<\lambda<b, 0<\varepsilon<1)$. Also 
we can easily see by Theorem 2 and Lebesgue's convergence theorem that the second and the third terms converge in $L_{2,\left(1+\varepsilon_{0}\right) / 2}$ as $\varepsilon \rightarrow 0$, and the limits are continuous functions of $\lambda>0$ in $L_{2,\left(1+\varepsilon_{0}\right) / 2}$.

We have by (2.1) of $\S 2, \nabla X=0$ for $|x|<1$ and $|\nabla X(x, \operatorname{Re} \sqrt{\lambda \pm i \varepsilon})|$ $\leq C(1+|x|)^{-\delta}$ where the constant $C$ does not depend on $\lambda, \varepsilon(a<\lambda<b$, $0<\varepsilon<1)$. Hence by Lemma 2.8 we have the following inequalities concerning the fourth and the fifth terms:

$$
\begin{aligned}
& \left\|\frac{\partial X}{\partial r}\left(\frac{\partial}{\partial r}+\frac{n-1}{2 r} \mp i \sqrt{ } \lambda\right)\left(U_{ \pm}^{*}(\lambda, \varepsilon) R_{2}(\lambda \pm i \varepsilon) f\right)\right\|_{\left(1+\varepsilon_{0}\right) / 2} \\
& \leq C\|f\|_{\left(3-\varepsilon_{0}\right) / 2}, \\
& \left\|\widetilde{\operatorname{grad}} X \cdot \widetilde{\operatorname{grad}}\left(U_{ \pm}^{*}(\lambda, \varepsilon) R_{2}(\lambda \pm i \varepsilon) f\right)\right\|_{\left(1+\varepsilon_{0}\right) / 2} \leq C\|f\|_{\left(3-\varepsilon_{0}\right) / 2} \text {. }
\end{aligned}
$$

Also it is easy to see that the fourth and the fifth terms converge in $L_{2,\left(1+\varepsilon_{0}\right) / 2}$ and the limits are strongly continuous function of $\lambda>0$ in $L_{2,\left(1+\varepsilon_{0}\right) / 2}$ by Lemma 2.8 .

The sixth term can be treated in the same way by Lemma 2.4 and Lemma 2.6.

Next we consider $G_{21}(\lambda \pm i \varepsilon) f$. We have to note that the assertion of Lemma 2.8 is also true for $R_{1}(\lambda \pm i \varepsilon) f$ in place of $v(\kappa: f)=e^{i X\left(x, \kappa_{1}\right)}$ $\times R_{2}\left(\kappa^{2}\right) f$, because in this case we can take $X \equiv 0$. So, we can treat $G_{21}(\lambda \pm i \varepsilon) f$ in a similar way to $G_{12}(\lambda \pm i \varepsilon) f$. Hence by Theorem 1 of $\S 1$, we can complete the proof. $\quad$ Q.E.D.

\section{§4. Eigenfunction Expansions and the Stationary Wave Operators}

In this section, we consider the relation between our stationary wave operator and the eigenfunction expansion theory developed by Saitō [12].

First we introduce some notations.

$$
\begin{aligned}
& \mathscr{D}_{ \pm r}=\frac{\partial}{\partial r}+\frac{n-1}{2 r} \mp i \sqrt{\lambda} \quad(r=|x|) . \\
& \mathscr{D}_{ \pm}=\operatorname{grad}+\frac{n-1}{2 r} \widetilde{x} \mp i \sqrt{\lambda} \widetilde{x} .
\end{aligned}
$$




$$
\begin{aligned}
& S_{\rho}=\left\{x \in \mathbb{R}^{n}:|x|=\rho\right\} . \\
& S^{n-1}: \text { the unit sphere in } \mathbb{R}^{n} .
\end{aligned}
$$

Let us consider $H_{2}=-\Delta+V(x)$, where $V(x)$ satisfies the condition (C) in $\S 2$. Consider the solution

$$
u(\lambda \pm i 0: f)=R_{2}(\lambda \pm i 0) f
$$

of $\left(H_{2}-\lambda\right) u=f\left(f \in L_{2,\left(3-\varepsilon_{0}\right) / 2}, \lambda>0\right)$ satisfying the radiation condition

$$
\left\|\mathscr{D}_{ \pm} u(\lambda \pm i 0: f)\right\|_{-\left(1-\varepsilon_{0}\right) / 2, E_{1}}<\infty .
$$

The following Lemma 4.1 plays a crucial role in the eigenfunction expansion.

Lemma 4. 1. ([11]). Let $f \in L_{2,\left(3-\varepsilon_{0}\right) / 2}$. (1) There exists a sequence $\left\{r_{m}\right\}$ of positive numbers diverging to infinity such that for $m \rightarrow \infty$

$$
\begin{aligned}
& r_{m}^{-\varepsilon_{0}} \int_{S_{r_{m}}}|u(\lambda \pm i 0: f)|^{2} d S \rightarrow 0, \\
& r_{m}^{2-\varepsilon_{0}} \int_{S_{r_{m}}}\left|\mathscr{D}_{ \pm}\left(e^{i x} u(\lambda \pm i 0: f)\right)\right|^{2} d S \rightarrow 0,
\end{aligned}
$$

where $X$ is the same as in $\S 2, X=X(x, \pm \sqrt{\lambda})$.

(2) There exists a strong limit

$$
{\mathrm{s}-\lim _{m \rightarrow \infty}}_{r_{m}^{(n-1) / 2}} \exp \left(\mp i \sqrt{ } \lambda r_{m}+i X\left(r_{m} \cdot, \pm \sqrt{\lambda}\right)\right)(u(\lambda \pm i 0: f))\left(r_{m} \cdot\right)
$$

in $L_{2}\left(S^{n-1}\right)$, where $\left\{r_{m}\right\}$ is any sequence specified in (1). This limit is independent of the choice of $\left\{r_{m}\right\}$.

Then, the following definition makes sense.

Definition 4. 2. For $\lambda>0$, and $f \in L_{2,\left(3-\varepsilon_{0}\right) / 2}$ let $\mathscr{F}_{2 \pm}(\lambda): L_{2,\left(3-\varepsilon_{0}\right) / 2}$ $\rightarrow L_{2}\left(S^{n-1}\right)$ be defined by

$$
\mathcal{F}_{2 \pm}(\lambda) f=\pi^{-1 / 2} \lambda^{1 / 4} \operatorname{sim}_{m \rightarrow \infty} r_{m}^{(n-1) / 2} e^{i \theta_{z}\left(r_{m}, \lambda\right)}\left(R_{2}(\lambda \pm i 0) f\right)\left(r_{m} \cdot\right),
$$

where $\theta_{ \pm}\left(r_{m}, \lambda\right)=\mp \sqrt{\lambda} r_{m}+X\left(r_{m} \cdot, \pm \sqrt{\lambda}\right)$, and $\left\{r_{m}\right\}$ is any sequence specified in (1) of Lemma 4.1. 
Now, we can state the result of [12].

Theorem 4. ([12]). (a) For $f, g \in L_{2,\left(3-\varepsilon_{0}\right) / 2}, \lambda>0$, the follorving relations hold:

$$
\begin{aligned}
& \mathscr{F}_{2 \pm}(\lambda) \in \boldsymbol{B}\left(L_{2,\left(3-\varepsilon_{0}\right) / 2}: L_{2}\left(S^{n-1}\right)\right) \\
& \left(\mathcal{F}_{2 \pm}(\lambda) f, \mathscr{F}_{2 \pm}(\lambda) g\right)_{L_{2}\left(S^{n-1}\right)} \\
& \quad=\frac{1}{2 \pi i}\left(R_{2}(\lambda+i 0) f-R_{2}(\lambda-i 0) f, g\right) .
\end{aligned}
$$

(b) Let $\mathscr{F}_{2 \pm}$ be defined by $\left(\mathscr{F}_{2 \pm} f\right)(\lambda)=\mathscr{F}_{2 \pm}(\lambda) f$; and let $\widehat{\mathcal{H}}=L_{2}$ $\times\left((0, \infty): L_{2}\left(S^{n-1}\right)\right)$ be the Hilbert space of all $L_{2}\left(S^{n-1}\right)$-valued square integrable functions over $(0, \infty)$. We have $\mathscr{F}_{2_{ \pm}} \in \boldsymbol{B}\left(L_{2,\left(3-\varepsilon_{0}\right) / 2}: \widehat{\mathcal{H}}\right)$. Moreover $\mathscr{F}_{2 \pm}$ can be uniquely extended to a partial isometry from $\mathcal{H}$ onto $\widehat{\mathcal{H}}$ with the initial set $E_{2, a c} \mathcal{H}$ (the absolutely continuous subspace for $\left.H_{2}\right)$, which will be denoted by $\mathscr{F}_{2 \pm}$. $\left(\mathcal{H}_{2 \pm}=L_{2}\left(\boldsymbol{R}^{n}\right)\right)$.

(c) For $f, g \in E_{2, a c} \mathcal{H}$ and any bounded Borel function $\alpha(\lambda)$ defined on the real line, we have

$$
\begin{aligned}
\left(\alpha\left(H_{2}\right) f, g\right) & =\int_{0}^{\infty} \alpha(\lambda)\left(\left(\mathscr{F}_{2 \pm} f\right)(\lambda),\left(\mathscr{F}_{2 \pm} g\right)(\lambda)\right)_{L_{2}\left(S^{n-1}\right)} d \lambda \\
& =\left(\alpha \mathscr{F}_{2 \pm} f, \mathscr{F}_{{ }_{2}} g\right)_{\hat{\jmath}},
\end{aligned}
$$

where by $\alpha$ is meant the operator of multiplication by the function $\alpha(\lambda)$. (This is a diagonal representation of $H_{2}$ ).

(d) The inversion formula holds for an arbitrary $f \in E_{2, a c} \mathcal{H}$ :

$$
f=\operatorname{s-lim} \int_{N \rightarrow \infty}^{N} \mathscr{F}_{2 \pm}(\lambda) *\left(\mathscr{F}_{2 \pm} f\right)(\lambda) d \lambda .
$$

In the same way, we can define $\mathscr{F}_{1 \pm}$ for $H_{1}=-\Delta$. (In this case we take $X=0$ ).

Let us take a Borel set $e$ contained in $(a, b) \quad(0<a<b<\infty)$ and let $\chi_{e}(\lambda)$ be the characteristic function of $e$. We can define a stationary wave operator which is "formally" different from $W_{j k}^{ \pm}(e)$ we have discussed in $\S 3$.

Definition 4. 3. Let $\Omega_{21}^{ \pm}(e)$ be defined by

$$
\Omega_{21}^{ \pm}(e)=\mathscr{F}_{2 \pm}^{*} \chi_{e} \mathscr{F}_{1 \pm},
$$


where $\chi_{e}$ denotes the operator of multiplication by $\chi_{e}(\lambda)$ in $\widehat{\mathcal{H}}$.

The purpose of this section is to prove the following theorem.

Theorem 5. $\quad W_{21}^{ \pm}(e)=\Omega_{21}^{ \pm}(e)$.

Proof. It suffices to show the following equality

$$
\left(E_{2}^{\prime}(\lambda) G_{21}(\lambda \pm i 0) g, f\right)=\left(\mathscr{F}_{1 \pm}(\lambda) g, \mathscr{F}_{2 \pm}(\lambda) f\right)_{L_{2}\left(S^{n-1}\right)}
$$

for arbitrary $f, g \in L_{2,\left(3-\varepsilon_{0}\right) / 2}$. Indeed, if (4.2) has been established, we have only to integrate both sides with respect to $\lambda$ on $e$. Then we have

$$
\left(W_{21}^{ \pm}(e) g, f\right)=\left(\chi_{e} \mathscr{F}_{1 \pm} g, \mathscr{F}_{2 \pm} f\right)_{\widehat{\leftrightarrow}}=\left(\mathscr{F}_{2 \pm}^{*} \chi_{e} \mathscr{F}_{1 \pm} g, f\right) .
$$

Taking into account that $L_{2,\left(3-\varepsilon_{0}\right) / 2}$ is dense in $\mathscr{H}$, we can conclude that $W_{21}^{ \pm}(e)=\mathscr{F}_{2 \pm}^{*} \chi_{e} \mathscr{F}_{1 \pm}$.

Now, let us prove (4.2). Let $u=U_{ \pm}^{*}(\lambda, 0) R_{2}(\lambda \pm i 0) f, v=R_{1}(\lambda \pm i 0) g$, $w=R_{1}(\lambda \mp i 0) g$. First we note the following lemma.

Lemma 4. 4. There exists a sequence $\left\{r_{m}\right\}$ tending to $\infty$ such that for $m \rightarrow \infty$

$$
\begin{aligned}
& r_{m}^{-\varepsilon_{0}} \int_{S_{r_{m}}}|u(x)|^{2} d S \rightarrow 0, \quad r_{m}^{2-\varepsilon_{0}} \int_{S_{r_{m}}}\left|\mathscr{D}_{ \pm} u\right|^{2} d S \rightarrow 0, \\
& r_{m}^{-\varepsilon_{0}} \int_{S_{r_{m}}}|v(x)|^{2} d S \rightarrow 0, \quad r_{m}^{2-\varepsilon_{0}} \int_{S_{r_{m}}}\left|\mathscr{D}_{ \pm} v\right|^{2} d S \rightarrow 0, \\
& r_{m}^{-\varepsilon_{0}} \int_{S_{r_{m}}}|w(x)|^{2} d S \rightarrow 0, \quad r_{m}^{2-\varepsilon_{0}} \int_{S_{r_{m}}}\left|\mathscr{D}_{\mp} w\right|^{2} d S \rightarrow 0,
\end{aligned}
$$

hold.

Proof. We have by assumption and Lemma 2.2

$$
\begin{aligned}
\int_{E_{1}}\left\{(1+|x|)^{-\left(1+\varepsilon_{0}\right)}\left(|u|^{2}+|v|^{2}+|w|^{2}\right)\right. & \\
& \left.+(1+|x|)^{1-\varepsilon_{0}}\left(\left|\mathscr{D}_{ \pm} u\right|^{2}+\left|\mathscr{D}_{ \pm} v\right|^{2}+\left|\mathscr{D}_{\mp} w\right|^{2}\right)\right\} d x<\infty .
\end{aligned}
$$

Hence the assertion of the lemma readily follows. $\quad$ Q.E.D. 
Proof of Theorem 5 (continued).

By Green's formula we have

$$
\begin{aligned}
\int_{|x|<r_{m}}\{(\Delta v) \bar{u}-v(\overline{\Delta u})\} d x= & \int_{s_{r_{m}}}\left(\frac{\partial v}{\partial r} \bar{u}-v \frac{\overline{\partial u}}{\partial r}\right) d S \\
= & \int_{S_{r_{m}}}\left\{\left(\mathscr{D}_{ \pm r} v\right) \bar{u}-v\left(\overline{\mathscr{D}} \overline{ \pm}_{ \pm r} u\right)\right\} d S \\
& \pm 2 i \sqrt{\lambda} \int_{s_{l_{m}}} v \bar{u} d S,
\end{aligned}
$$

where $\left\{r_{m}\right\}$ is a sequence specified in Lemma 4.4. Noting that

$$
v \bar{u}=\exp (\mp i \sqrt{\lambda} r) R_{1}(\lambda \pm i 0) g \times \overline{\exp (\mp i \sqrt{\lambda} r+i X) R_{2}(\lambda \pm i 0) f},
$$

by Definition 4.2 and Lemma 4.4 we have,

$$
\begin{aligned}
\frac{1}{2 \pi i} \lim _{r_{m} \rightarrow \infty} \int_{|x|<r_{m}}\{(\Delta v) \bar{u}-v(\overline{\Delta u})\} d x \\
= \pm\left(\mathscr{F}_{1 \pm}(\lambda) g, \mathscr{F}_{2 \pm}(\lambda) f\right)_{L_{2}\left(S^{n-1}\right)} .
\end{aligned}
$$

Similarly by Green's formula

$$
\begin{aligned}
\int_{|x|<r_{m}}\{(\Delta w) \bar{u}-w(\overline{\Delta u})\} d x & =\int_{S_{r_{m}}}\left(\frac{\partial w}{\partial r} \bar{u}-w \frac{\overline{\partial u}}{\partial r}\right) d S \\
& =\int_{S_{r_{m}}}\left\{\left(\mathscr{D}_{\mp r} w\right) \bar{u}-w\left(\overline{\mathscr{D}_{ \pm r} u}\right)\right\} d S .
\end{aligned}
$$

Hence by Lemma 4.4, we have

$$
\frac{1}{2 \pi i} \lim _{r_{m} \rightarrow \infty} \int_{|x|<r_{m}}\{(\Delta w) \bar{u}-w(\overline{\Delta u})\} d x=0 .
$$

Let us compute the left hand side of (4.3) and (4.4). Introducing the function

$$
\chi_{m}=\left\{\begin{array}{lll}
1 & \text { if } & |x|<r_{m} \\
0 & \text { if } & |x|>r_{m},
\end{array}\right.
$$

and replacing $\Delta$ by $-\left(H_{1}-(\lambda \pm i \varepsilon)\right)-(\lambda \pm i \varepsilon)$, we have

$$
\begin{aligned}
& \left(\chi_{m} \Delta R_{1}(\lambda \pm i \varepsilon) g, U_{ \pm}^{*}(\lambda, \varepsilon) R_{2}(\lambda \pm i \varepsilon) f\right) \\
& \quad-\left(\chi_{m} R_{1}(\lambda \pm i \varepsilon) g, \Delta\left(U_{ \pm}^{*}(\lambda, \varepsilon) R_{2}(\lambda \pm i \varepsilon) f\right)\right)
\end{aligned}
$$




$$
\begin{gathered}
=\left(\chi_{m} R_{1}(\lambda \pm i \varepsilon) g, G_{12}(\lambda \pm i \varepsilon) f\right)-\left(\chi_{m} g, U_{ \pm}^{*}(\lambda, \varepsilon) R_{2}(\lambda \pm i \varepsilon) f\right) \\
\mp 2 i \varepsilon\left(\chi_{m} R_{1}(\lambda \pm i \varepsilon) g, U_{ \pm}^{*}(\lambda, \varepsilon) R_{2}(\lambda \pm i \varepsilon) f\right) .
\end{gathered}
$$

Letting $\varepsilon$ tend to 0 , we see $R_{1}(\lambda \pm i \varepsilon) g \rightarrow v$ in $H_{1 \mathrm{oc}}^{2}$ and $U_{ \pm}^{*}(\lambda, \varepsilon)$ $\times R_{2}(\lambda \pm i \varepsilon) f \rightarrow u$ in $H_{\text {loc. }}^{2}$ Hence we have

$$
\begin{aligned}
& \left(\chi_{m} \Delta v, u\right)-\left(\chi_{m} v, \Delta u\right) \\
& \quad=\left(\chi_{m} R_{1}(\lambda \pm i 0) g, G_{12}(\lambda \pm i 0) f\right)-\left(\chi_{m} g, U_{ \pm}^{*}(\lambda, 0) R_{2}(\lambda \pm i 0) f\right) .
\end{aligned}
$$

Similarly,

$$
\begin{aligned}
& \left(\chi_{m} \Delta w, u\right)-\left(\chi_{m} w, \Delta u\right) \\
& \quad=\left(\chi_{m} R_{1}(\lambda \mp i 0) g, G_{12}(\lambda \pm i 0) f\right)-\left(\chi_{m} g, U_{ \pm}^{*}(\lambda, 0) R_{2}(\lambda \pm i 0) f\right) .
\end{aligned}
$$

Subtracting (4.6) from (4.5), we have

$$
\text { (4.7) } \begin{gathered}
\frac{1}{2 \pi i} \int_{|x|<r_{m}}\{(\Delta v) \bar{u}-v(\overline{\Delta u})\} d x-\frac{1}{2 \pi i} \int_{\mid x_{\mid}<r_{m}}\{(\Delta w) \bar{u}-w(\overline{\Delta u})\} d x \\
= \pm\left(\chi_{m} \frac{1}{2 \pi i}\left[R_{1}(\lambda+i 0)-R_{1}(\lambda-i 0)\right] g, G_{12}(\lambda \pm i 0) f\right) .
\end{gathered}
$$

Letting $m$ tend to infinity, by (4.3), (4.4) and (4.7), we have

$$
\left(E_{1}^{\prime}(\lambda) g, G_{12}(\lambda \pm i 0) f\right)=\left(\mathscr{F}_{1 \pm}(\lambda) g, \mathscr{F}_{2 \pm}(\lambda) f\right)_{L_{2}\left(S^{n-1}\right)} .
$$

By (1.7) of $\S 1$, the left hand side of (4.8) is equal to $\left(E_{2}^{\prime}(\lambda)\right.$ $\left.\times G_{21}(\lambda \pm i 0) g, f\right)$, which proves (4.2).

Q.E.D.

\section{$\S 5$. Remarks on the Short-Range Perturbation}

In this section we consider the case in which $V(x)$ has a shortrange part. More precisely, we assume the following condition:

(C) $" \quad V(x)=V_{L}(x)+V_{S}(x)$, where $V_{L}(x)$ satisfies the condition

(C) in $\S 2$, and $V_{S}(x)$ is a bounded real function having the following decaying order

$$
V_{S}(x)=O\left(|x|^{-1-8}\right) \text { as } \quad|x| \rightarrow \infty .
$$

We denote by $H_{3}$ the unique self-adjoint extension of $-\Delta+V_{L}(x)$ $+V_{S}(x)$ restricted to $C_{0}^{\infty}\left(\boldsymbol{R}^{n}\right)$. Also we set $H_{1}=-\Delta, H_{2}=-\Delta+V_{L}(x)$. 
It seems that the general theory of $\S 1$ cannot be applied directly to $H_{1}$ and $H_{3}$, because Lemma 2.2, which is crucial to see that the assumption (A-2) is satisfied, cannot be proved without assuming the differentiability on $V_{S}(x)$. So we construct the stationary wave operator, which shows the similarity of $H_{1}$ and $H_{3}$, using the so called "chain rule".

First let us prove the following theorem.

Theorem 6. Let $e$ be a Borel set in $(a, b)(0<a<b<\infty)$. There exist stationary wave operators $W_{32}^{ \pm}(e), W_{23}^{ \pm}(e)$ having the following properties.

(1) $W_{j k}^{ \pm}(e)$ is a partial isometry with the initial set $E_{k}(e) \mathcal{H}$ and the final set $E_{j}(e) \mathcal{H}(j, k=2,3)$.

(2) $\quad\left(W_{j k}^{ \pm}(e)\right)^{*}=W_{k j}^{ \pm}(e) \quad(j, k=2,3)$.

(3) $H_{j} W_{j k}^{ \pm}(e) \supseteq W_{j k}^{ \pm}(e) H_{k}(j, k=2,3)$.

Proof. We first note that Theorem 2 is also true for $H_{3}=-\Delta$ $+V_{L}(x)+V_{S}(x)$ (see Ikebe-Saitō [7]). Next in $\S 1$ we take the identity operator as $U_{ \pm}(\lambda, \varepsilon)$, and $\mathscr{H}_{+}=\widetilde{\mathscr{H}}_{+}=L_{2,\left(1+\varepsilon_{0}\right) / 2}, \mathscr{H}_{-}=L_{2,-\left(1+\varepsilon_{0}\right) / 2}$. By direct calculation we have for $f \in L_{2,\left(1+\varepsilon_{0}\right) / 2}$

$$
\begin{aligned}
& G_{32}(\lambda \pm i \varepsilon) f=\left(H_{3}-(\lambda \pm i \varepsilon)\right) R_{2}(\lambda \pm i \varepsilon) f=f+V_{S}(x) R_{2}(\lambda \pm i \varepsilon) f, \\
& G_{23}(\lambda \pm i \varepsilon) f=\left(H_{2}-(\lambda \pm i \varepsilon)\right) R_{3}(\lambda \pm i \varepsilon) f=f-V_{S}(x) R_{3}(\lambda \pm i \varepsilon) f,
\end{aligned}
$$

By Theorem 2, $R_{2}(\lambda \pm i \varepsilon) f, R_{3}(\lambda \pm i \varepsilon) f$ converge in $L_{2,-\left(1+\varepsilon_{0}\right) / 2}$ as $\varepsilon$ tend to 0 . Taking into account that $V_{S}(x)=O\left(|x|^{-1-\delta}\right)$, we can see that the assumption (A-2) of $\S 1$ is satisfied. So, by Theorem 1 , we can construct the stationary wave operators $W_{32}^{ \pm}(e), W_{23}^{ \pm}(e)$ having the properties stated above.

Q.E.D.

We have already constructed the stationary wave operators $W_{21}^{ \pm}(e)$, $W_{12}^{ \pm}(e)$ which shows the similarity of $H_{1}$ and $H_{2}$ in $\S 3$. Now, define $W_{31}^{ \pm}(e), W_{13}^{ \pm}(e)$ by

$$
\begin{aligned}
& W_{31}^{ \pm}(e)=W_{32}^{ \pm}(e) W_{21}^{ \pm}(e), \\
& W_{13}^{ \pm}(e)=W_{12}^{ \pm}(e) W_{23}^{ \pm}(e) .
\end{aligned}
$$


Then it is easy to prove the following theorem, in view of Theorem 3 and Theorem 6.

Theorem 7. $W_{31}^{ \pm}(e), W_{13}^{ \pm}(e)$, defined above, have the following properties.

(1) $W_{j k}^{ \pm}(e)$ is a partial isometry with the initial set $E_{k}(e) \mathcal{H}$ and the final set $E_{j}(e) \mathcal{H}$.

(2) $\quad\left(W_{j k}^{+}(e)\right)^{*}=W_{k j}^{ \pm}(e)$.

(3) $\quad H_{j} W_{j k}^{ \pm}(e) \supseteq W_{j k}^{ \pm}(e) H_{k}(j, k=1,3)$.

Next let us outline the idea of Ikebe [5] concerning the short-range perturbation of the eigenfunction expansion. In $\S 4$, we have already explained the construction of the operator $\mathscr{F}_{2 \pm}(\lambda) \in \boldsymbol{B}\left(L_{2,\left(3-\varepsilon_{0}\right) / 2}: L_{2}\left(S^{n-1}\right)\right)$ associated with $H_{2}=-\Delta+V_{L}(x)$. But by Theorem 2 and the formula (4. 1)

$$
\left(\mathscr{F}_{2 \pm}(\lambda) f, \mathscr{F}_{2 \pm}(\lambda) g\right)_{L_{2}\left(S^{n-1}\right)}=\frac{1}{2 \pi i}\left(R_{2}(\lambda+i 0) f-R_{2}(\lambda-i 0) f, g\right),
$$

which is valid for $f, g \in L_{2,\left(3-\varepsilon_{0}\right) / 2}$, we can uniquely extend $\mathscr{F}_{2 \pm}(\lambda)$ as a bounded operator from $L_{2,\left(1+\varepsilon_{0}\right) / 2}$ to $L_{2}\left(S^{n-1}\right)$. We use the same notation for this extension. Since $V_{\boldsymbol{S}} \in \boldsymbol{B}\left(L_{2,-\left(1+\varepsilon_{0}\right) / 2}: L_{2,\left(1+\varepsilon_{0}\right) / 2}\right)$, which is easily seen by the condition (C)", the following definition makes sense.

Definition 5. 1. $\mathscr{F}_{3 \pm}(\lambda)=\mathscr{F}_{2 \pm}(\lambda)\left(1-V_{S} R_{3}(\lambda \pm i 0)\right)$ for $\lambda>0$.

When we set $\left(\mathscr{F}_{3 \pm} f\right)(\lambda)=\mathscr{F}_{3 \pm}(\lambda) f$ for $f \in L_{2,\left(1+\varepsilon_{0}\right) / 2}, \mathscr{F}_{3_{ \pm}}$can be uniquely extended to a partial isometry on $\mathcal{H}$ with the initial set $E_{3, a c} \mathscr{H}$ (the absolutely continuous subspace for $\left.H_{3}\right)$ and the final set $L_{2}((0, \infty)$ : $\left.L_{2}\left(S^{n-1}\right)\right)$, where we also use the same notation for the extended operator. Then we can get the spectral representation associated with $H_{3}$ with the aid of $\mathscr{F}_{3 \pm}$. See for the details Ikebe [5].

Now, we can define the stationary wave operator by the spectral representation as in $\S 4$.

Definition 5. 2. Let $\Omega_{32}^{ \pm}(e), \Omega_{31}^{ \pm}(e)$ be defined by 


$$
\begin{aligned}
& \Omega_{32}^{ \pm}(e)=\mathscr{F}_{3 \pm}^{*} \chi_{e} \mathscr{F}_{2 \pm}, \\
& \Omega_{31}^{ \pm}(e)=\mathscr{F}_{3 \pm}^{*} \chi_{e} \mathscr{F}_{1 \pm},
\end{aligned}
$$

where by $\chi_{e}$ is meant the operator of multiplication by the function $\chi_{e}(\lambda)$.

Then we can prove the following theorem.

Theorem 8. $\quad W_{32}^{ \pm}(e)=\Omega_{32}^{ \pm}(e)$.

Proof. As in the proof of Theorem 5, we have only to prove the following equality:

$$
\left(E_{3}{ }^{\prime}(\lambda) G_{32}(\lambda \pm i 0) f, g\right)=\left(\mathscr{F}_{2 \pm}(\lambda) f, \mathscr{F}_{3 \pm}(\lambda) g\right)_{L_{2}\left(S^{n-1}\right)}
$$

for $f, g \in L_{2,\left(1+\varepsilon_{0}\right) / 2}, \lambda>0$.

By the resolvent equation we have

$$
\begin{aligned}
& \left(\frac{1}{2 \pi i}\left[R_{3}(\lambda+i \varepsilon)-R_{3}(\lambda-i \varepsilon)\right] G_{32}(\lambda \pm i \varepsilon) f, g\right) \\
& =\left(\frac{\varepsilon}{\pi} R_{3}(\lambda+i \varepsilon) R_{3}(\lambda-i \varepsilon)\left(H_{3}-(\lambda \pm i \varepsilon)\right) R_{2}(\lambda \pm i \varepsilon) f, g\right) \\
& =\left(\frac{\varepsilon}{\pi} R_{3}(\lambda \mp i \varepsilon) R_{2}(\lambda \pm i \varepsilon) f, g\right) \\
& =\left(\frac{\varepsilon}{\pi} R_{2}(\lambda \mp i \varepsilon) R_{2}(\lambda \pm i \varepsilon) f, g\right) \\
& \quad-\left(\frac{\varepsilon}{\pi} R_{3}(\lambda \mp i \varepsilon) V_{S} R_{2}(\lambda \mp i \varepsilon) R_{2}(\lambda \pm i \varepsilon) f, g\right) \\
& =\left(\frac{1}{2 \pi i}\left[R_{2}(\lambda+i \varepsilon)-R_{2}(\lambda-i \varepsilon)\right] f, g\right) \\
& \quad-\left(\frac{1}{2 \pi i}\left[R_{2}(\lambda+i \varepsilon)-R_{2}(\lambda-i \varepsilon)\right] f, V_{S} R_{3}(\lambda \pm i \varepsilon) g\right) .
\end{aligned}
$$

Letting $\varepsilon$ tend to 0 , we see by Theorem 2

$$
\begin{array}{r}
\left(E_{3}{ }^{\prime}(\lambda) G_{32}(\lambda \pm i 0) f, g\right)=\left(\frac{1}{2 \pi i}\left[R_{2}(\lambda+i 0)-R_{2}(\lambda-i 0)\right] f, g\right) \\
-\left(\frac{1}{2 \pi i}\left[R_{2}(\lambda+i 0)-R_{2}(\lambda-i 0)\right] f, V_{S} R_{3}(\lambda \pm i 0) g\right) .
\end{array}
$$


But by (4.1) of $\S 4$ and Definition 5.1, the right hand side of this equality is rewritten as

$$
\begin{aligned}
\left(\mathscr{F}_{2 \pm}(\lambda) f, \mathscr{F}_{2 \pm}(\lambda) g\right)_{L_{2}\left(S^{n-1}\right)}-\left(\mathscr{F}_{2 \pm}(\lambda) f, \mathscr{F}_{2 \pm}(\lambda) V_{S} R_{3}(\lambda \pm i 0) g\right)_{L_{2}\left(S^{n-1}\right)} \\
=\left(\mathscr{F}_{2 \pm}(\lambda) f, \mathscr{F}_{2 \pm}(\lambda)\left(1-V_{S} R_{3}(\lambda \pm i 0)\right) g\right)_{L_{2}\left(S^{n-1}\right)} \\
=\left(\mathscr{F}_{2 \pm}(\lambda) f, \mathscr{F}_{{ }^{2}}(\lambda) g\right)_{L_{2}\left(S^{n-1}\right)},
\end{aligned}
$$

which proves (5.3).

Q.E.D.

Since $\mathscr{F}_{2 \pm}$ is a unitary operator on $E_{2, a c} \mathcal{H}$ onto $L_{2}\left((0, \infty): L_{2}\left(S^{n-1}\right)\right)$, we have

$$
\begin{aligned}
\Omega_{32}^{ \pm}(e) \Omega_{21}^{ \pm}(e) & =\mathscr{F}_{3 \pm}^{*} \chi_{e} \mathscr{F}_{2 \pm} \mathscr{F}_{2 \pm}^{*} \chi_{e} \mathscr{F}_{1 \pm} \\
& =\mathscr{F}_{3 \pm}^{*} \chi_{e} \mathscr{F}_{1 \pm} \\
& =\Omega_{31}^{ \pm}(e) .
\end{aligned}
$$

By this fact and Theorems 5, 6, 7 and 8, we can easily get the following theorem.

Theorem 9. $\quad W_{31}^{ \pm}(e)=\Omega_{31}^{ \pm}(e)$.

\section{Appendix. An Estimate Concerning the Radiation Condition}

In this Appendix we prove Lemma 2.2.

Let $u \in C_{0}^{\infty}\left(\boldsymbol{R}^{n}\right)$ and let $f=\left(-\Delta+V(x)-\kappa^{2}\right) u, \kappa \in \bar{K}_{ \pm}$. We put $v=e^{i X} u$. Then $v$ satisfies the following equation,

$$
\begin{aligned}
-\Delta v & +\left(|\nabla X|^{2}+V(x)-2 \kappa \frac{\partial X}{\partial r}\right) v-\kappa^{2} v \\
= & e^{i x} f-i\left(\frac{\partial^{2} X}{\partial r^{2}}+\frac{\Lambda X}{r^{2}}\right) v-2 i \sum_{j} \frac{\partial X}{\partial x_{j}} \mathscr{D}_{j} v,
\end{aligned}
$$

where $\Lambda$ denotes the Laplace-Beltrami operator on the unit sphere. We can rewrite this equation as follows,

$$
\begin{gathered}
-\sum_{j} \frac{\partial}{\partial x_{j}} \mathscr{D}_{j} v+\frac{n-1}{2 r} \mathscr{D}_{r} v+\left(\widetilde{V}(x)+|\nabla X|^{2}-2 \kappa \frac{\partial X}{\partial r}\right) v-i \kappa \mathscr{D}_{r} v \\
=e^{i X} f-i\left(\frac{\partial^{2} X}{\partial r^{2}}+\frac{\Lambda X}{r^{2}}\right) v-2 i \sum_{j} \frac{\partial X}{\partial x_{j}} \mathscr{D}_{j} v,
\end{gathered}
$$


where $\widetilde{V}(x)=V(x)+\frac{(n-1)(n-3)}{4 r^{2}}$.

Let us put $\phi(x)=\alpha(|x|)(1+|x|)^{2 \beta+1}$, where $\beta=\left(1-\varepsilon_{0}\right) / 2$ and $\alpha(r)$ is a $C^{1}$-function on $(0, \infty)$ such that $0 \leq \alpha(r) \leq 1, \alpha^{\prime}(r)>0$ and

$$
\alpha(r)= \begin{cases}0 & (r<1) \\ 1 & (r>2) .\end{cases}
$$

Then we can prove the following identity.

\section{Proposition 1.}

$$
\begin{gathered}
\int \kappa_{2} \phi|\mathscr{D} v|^{2} d x+\int\left\{\left(\frac{\phi}{r}-\frac{1}{2} \frac{\partial \phi}{\partial r}\right)\left|\mathscr{D}_{v}\right|^{2}+\left(\frac{\partial \phi}{\partial r}-\frac{\phi}{r}\right)\left|\mathscr{D}_{r} v\right|^{2}\right\} d x \\
=\operatorname{Re} \int \phi e^{i X} f \overline{\mathscr{D}_{r} v} d x+\operatorname{Re} \int \phi\left(2 \kappa_{1} \frac{\partial X}{\partial r}-\widetilde{V(x)}-|\nabla X|^{2}\right) v \overline{\mathscr{D}_{r} v} d x \\
-\operatorname{Re} i \int \phi\left(\frac{\partial^{2} X}{\partial r^{2}}+\frac{\Lambda X}{r^{2}}\right) \overline{\mathscr{D}_{r} v} d x \\
-\operatorname{Re} 2 i \int \phi \sum_{j} \frac{\partial X}{\partial x_{j}} \mathscr{D}_{j} v \overline{\mathscr{D}_{r} v} d x \\
+\operatorname{Re} 2 \kappa_{2} i \int \phi v \frac{\partial X}{\partial r} \overline{\mathscr{D}_{r} v} d x .
\end{gathered}
$$

Proof. Let us multiply both sides (6.2) by $\phi \overline{\mathscr{D}_{r} v}$, and integrate by parts and take the real part. Then we can get this identity. (See for the details Ikebe-Saitō [7], Lemma 2.2.) Q.E.D.

Now, we shall estimate the last three terms of the right hand side of (6.3). For this purpose, we introduce other identities.

Proposition 2. For a real $C^{1}$-function $A(x)$, we have

$$
\begin{gathered}
\operatorname{Re} 2 i \int \phi A v \overline{\mathscr{D}_{r} v} d x=-\operatorname{Re} \frac{1}{\kappa_{1}} \int \frac{\partial \phi}{\partial r} A v \overline{\mathscr{D}_{r} v} d x \\
-\operatorname{Re} \frac{1}{\kappa_{1}} \int \phi v \sum_{j} \frac{\partial A}{\partial x_{j}} \overline{\mathscr{D}_{j} v} d x
\end{gathered}
$$




$$
\begin{aligned}
& -\frac{1}{\kappa_{1}} \int \phi A|\mathscr{D} v|^{2} d x \\
& +\frac{1}{\kappa_{1}} \int \phi A\left(2 \kappa_{1} \frac{\partial X}{\partial r}-V-|\nabla X|^{2}\right)|v|^{2} d x \\
& +\operatorname{Re} \frac{1}{\kappa_{1}} \int \phi A v e^{-i x} \bar{f} d x \\
& +\operatorname{Re} \frac{2 i}{\kappa_{1}} \int \phi A v \sum_{j} \frac{\partial X}{\partial x_{j}} \overline{\mathscr{D}_{j} v} d x .
\end{aligned}
$$

Proof. By (6.2), we have

(6.5) $\quad i \bar{\kappa} \overline{\mathscr{D}_{r} v}=\overline{\sum_{j} \frac{\partial}{\partial x_{j}} \mathscr{D}_{j} v}-\frac{n-1}{2 r} \overline{\mathscr{D}_{r} v}-\left(\widetilde{V(x)}+|\nabla X|^{2}-2 \bar{\kappa} \frac{\partial X}{\partial r}\right) \bar{v}$

$$
+e^{-i X} \bar{f}+i\left(\frac{\partial^{2} X}{\partial r^{2}}+\frac{\Lambda X}{r^{2}}\right) \bar{v}+2 i \sum_{j} \frac{\partial X}{\partial x_{j}} \overline{\mathscr{D}_{j} v} .
$$

Multiplying both sides by $\phi A v$ and integrating by parts give the following identity:

$$
\begin{aligned}
2 \kappa_{1} i \int \phi A v \overline{\mathscr{D}_{r} v} d x= & -\int \frac{\partial \phi}{\partial r} A v \overline{\mathscr{D}_{r} v} d x \\
& -\int \phi v \sum_{j} \frac{\partial A}{\partial x_{j}} \overline{\mathscr{D}_{j} v} d x \\
& -\int \phi A\left|\mathscr{D}_{v}\right|^{2} d x \\
& +\int \phi A\left(2 \kappa_{1} \frac{\partial X}{\partial r}-\widetilde{V}-|\nabla X|^{2}\right)|v|^{2} d x \\
& -2 \kappa_{2} i \int \phi A \frac{\partial X}{\partial r}|v|^{2} d x \\
& +\int \phi A v e^{-i X} \bar{f} d x \\
& +i \int \phi A\left(\frac{\partial^{2} X}{\partial r^{2}}+\frac{\Lambda X}{r^{2}}\right)|v|^{2} d x \\
& +2 i \int \phi A v \sum_{j} \frac{\partial X}{\partial x_{j}} \overline{\mathscr{D}_{j} v} d x .
\end{aligned}
$$

Let us take the real part of this identity. Then (6.4) follows.

Q.E.D. 
Now, we use the notation $[J]$ for the sum of the any of the following integrals:

$$
\left\{\begin{array}{l}
\int_{|x|>1} O\left(|x|^{2 \beta-2-\delta}\right)|v|^{2} d x, \int_{|x|>1} O\left(|x|^{2 \beta-\delta}\right) v \bar{f} d x \\
\int_{|x|>1} O\left(|x|^{2 \beta-\delta}\right)|\mathscr{D} v|^{2} d x, \quad \int_{|x|>1} O\left(|x|^{2 \beta-1-\delta}\right) v \overline{\mathcal{D}_{j} v} d x \\
\int_{|x|>1} O\left(|x|^{2 \beta+1-\delta}\right) \mathscr{D}_{j} v \bar{f} d x,
\end{array}\right.
$$

where $O\left(|x|^{d}\right)$ means a function which behaves like $|x|^{d}$ as $|x| \rightarrow \infty$.

\section{Proposition 3.}

$$
\begin{aligned}
& \operatorname{Re} i \int \phi\left(\frac{\partial^{2} X}{\partial r^{2}}+\frac{\Lambda X}{r^{2}}\right) v \overline{\mathscr{D}_{r} v} d x \\
& \quad=\operatorname{Re} \frac{i}{\kappa_{1}} \int \phi\left(\frac{\partial^{2} X}{\partial r^{2}}+\frac{\Lambda X}{r^{2}}\right) v \sum_{j} \frac{\partial X}{\partial x_{j}} \overline{\mathscr{D}_{j} v} d x+[J]
\end{aligned}
$$

Proof. Let us note that

$$
\begin{aligned}
& \frac{\partial^{2} X}{\partial r^{2}}+\frac{\Lambda X}{r^{2}}=O\left(r^{-1-\delta}\right), \\
& \nabla\left(\frac{\partial^{2} X}{\partial r^{2}}+\frac{\Lambda X}{r^{2}}\right)=O\left(r^{-2-\delta}\right) \quad(r=|x|) .
\end{aligned}
$$

Then (6.6) follows from (6.4), if we put $A=\frac{\partial^{2} X}{\partial r^{2}}+\frac{\Lambda X}{r^{2}}$.

Q.E.D.

\section{Proposition 4.}

$$
\begin{aligned}
\operatorname{Re} 2 i \int \phi \sum_{j} & \frac{\partial X}{\partial x_{j}} \mathscr{D}_{j} v \overline{\mathscr{D}_{r} v} d x \\
= & \operatorname{Re} \frac{i}{\kappa_{1}} \int \phi \sum_{j} \frac{\partial X}{\partial x_{j}} \mathscr{D}_{j} v\left(\frac{\partial^{2} X}{\partial r^{2}}+\frac{\Lambda X}{r^{2}}\right) \bar{v} d x \\
& -\operatorname{Re} 2 \frac{\kappa_{2}}{\kappa_{1}} i \int \phi \sum_{j} \frac{\partial X}{\partial x_{j}} \mathscr{D}_{j} v \frac{\partial X}{\partial r} \bar{v} d x \\
& -\frac{\kappa_{2}}{\kappa_{1}} \int \phi \frac{\partial X}{\partial r}\left|\mathscr{D}_{v}\right|^{2} d x+[J] .
\end{aligned}
$$


Proof. Let us multiply both sides of (6.5) by $\phi \sum_{j} \frac{\partial X}{\partial x_{j}} \mathscr{D}_{j} v$, integrate over $\boldsymbol{R}^{n}$, and take the real part. We have then

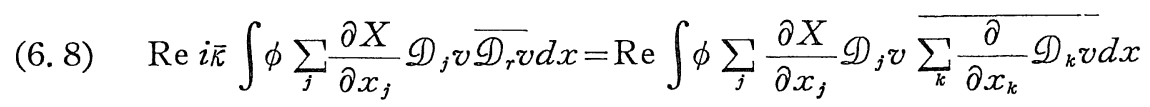

$$
\begin{aligned}
& -\operatorname{Re} 2 \kappa_{2} i \int \phi \sum_{j} \frac{\partial X}{\partial x_{j}} \mathscr{D}_{j} v \frac{\partial X}{\partial r} \bar{v} d x \\
& +\operatorname{Re} i \int \phi \sum_{j} \frac{\partial X}{\partial x_{j}} \mathscr{D}_{j} v\left(\frac{\partial^{2} X}{\partial r^{2}}+\frac{\Lambda X}{r^{2}}\right) \bar{v} d x+[J] .
\end{aligned}
$$

Integrating by parts and using the relation

$$
\frac{\partial}{\partial x_{k}} \mathscr{D}_{j} v=\frac{\partial}{\partial x_{j}} \mathscr{D}_{k} v+\left(\frac{n-1}{2 r}-i \kappa\right)\left(\widetilde{x}_{j} \mathscr{D}_{k} v-\widetilde{x}_{k} \mathscr{D}_{j} v\right),
$$

we have the following expression for the first term of the right hand side of (6.8):

$$
\begin{aligned}
\operatorname{Re} & \int \phi \sum_{j} \frac{\partial X}{\partial x_{j}} \mathscr{D}_{j} v \sum_{k} \frac{\partial}{\partial x_{k}} \mathscr{D}_{k} v d x \\
& =-\operatorname{Re} i \kappa \int \phi \sum_{j} \frac{\partial X}{\partial x_{j}} \mathscr{D}_{j} v \overline{\mathscr{D}_{r} v} d x-\kappa_{2} \int \phi \frac{\partial X}{\partial r}\left|\mathscr{D}_{v}\right|^{2} d x+[J] .
\end{aligned}
$$

Combining (6.8) and (6.9), we can see that (6.7) holds. Q.E.D.

Now, we can estimate the right hand side of (6.3).

Proposition 5. The following inequality holds:

$$
\begin{aligned}
\|\mathscr{D} v\|_{\beta, E_{2}}^{2} & \leq C\left(\|v\|_{\beta-1}^{2}+\|f\|_{\beta+1}^{2}+\|\mathscr{D} v\|_{\beta-\delta / 2, E_{1}}^{2}\right. \\
& +\kappa_{2}\|v\|_{\beta-\delta}\|v\|_{\beta-1}+\kappa_{2}\|v\|_{\beta-\delta}\|f\|_{\beta+1} \\
& \left.+\kappa_{2}\|v\|_{\beta-\delta / 2}\left\|\mathscr{D}_{v}\right\|_{\beta-\delta / 2, E_{1}}+\left\|\mathscr{D}_{v}\right\|_{\beta, B_{1}, \mathrm{z}}^{2}\right),
\end{aligned}
$$

where $C$ is a constanl which docs not depend on $u \in C_{0}^{\infty}\left(\mathbb{R}^{n}\right)$ and $\kappa \in \bar{K}_{ \pm}$.

Proof. Let us denote the $j$-th term of the right hand side of (6.3) by $I_{j}$. By Propositions 3 and 4 , we have

$$
I_{3}+I_{4}=\operatorname{Re} 2 \frac{\kappa_{2}}{\kappa_{1}} i \int \phi \sum_{j} \frac{\partial X}{\partial x_{j}} \mathscr{D}_{j} v \frac{\partial X}{\partial r} \bar{v} d x
$$




$$
+\operatorname{Re} \frac{\kappa_{2}}{\kappa_{1}} \int \phi \frac{\partial X}{\partial r}|D v|^{2} d x+[J]
$$

Hence, we have

$$
\begin{aligned}
I_{3}+I_{4}+I_{5} & =[J]+\kappa_{2}\left\{\operatorname{Re} 2 i \int \phi v \frac{\partial X}{\partial r} \overline{D_{r} v} d x\right. \\
& \left.+\operatorname{Re} \frac{2 i}{\kappa_{1}} \int \phi \sum_{j} \frac{\partial X}{\partial x_{j}} \mathscr{D}_{j} v \frac{\partial X}{\partial r} \bar{v} d x+\frac{1}{\kappa_{1}} \int \phi \frac{\partial X}{\partial r}\left|\mathscr{D}_{v}\right|^{2} d x\right\}
\end{aligned}
$$

We denote the right hand side of this equality by $[J]+I_{7}$. Let us put $A=\frac{\partial X}{\partial r}$ in (6.4). Then we have

$$
\begin{aligned}
I_{7}=\kappa_{2}\{ & -\operatorname{Re} \frac{1}{\kappa_{1}} \int \frac{\partial \phi}{\partial r} \frac{\partial X}{\partial r} v \overline{\mathscr{D}_{r} v} d x \\
& -\operatorname{Re} \frac{1}{\kappa_{1}} \int \phi v \sum_{j}\left(\frac{\partial}{\partial x_{j}} \frac{\partial X}{\partial r}\right) \overline{\mathscr{D}_{j} v} d x \\
& +\frac{1}{\kappa_{1}} \int \phi \frac{\partial X}{\partial r}\left(2 \kappa_{1} \frac{\partial X}{\partial r}-\widetilde{V}-|\nabla X|^{2}\right)|v|^{2} d x \\
& \left.+\operatorname{Re} \frac{1}{\kappa_{1}} \int \phi \frac{\partial X}{\partial r} v e^{-i x} \bar{f} d x\right\} .
\end{aligned}
$$

Hence by Schwarz' inequality, we can estimate $[J]+I_{7}$ from above as follows :

$$
\begin{aligned}
{[J]+I_{7} } & \leq C\left(\|v\|_{\beta-1}^{2}+\|f\|_{\beta+1}^{2}+\|\mathscr{D} v\|_{\beta-\delta / 2, E_{1}}^{2}\right. \\
& +\kappa_{2}\|v\|_{\beta-\delta}\|v\|_{\beta-1}+\kappa_{2}\|v\|_{\beta-\delta}\|f\|_{\beta+1} \\
& \left.+\kappa_{2}\|v\|_{\beta-\delta / 2}\|\mathscr{D} v\|_{\beta-\delta / 2, E_{1}}\right)
\end{aligned}
$$

By our assumption we have for $r>2$

$$
\begin{aligned}
& \frac{\phi}{r}-\frac{1}{2} \frac{\partial \phi}{\partial r}=\frac{\varepsilon_{0}}{2}(1+r)^{2 \beta}, \\
& \frac{\partial \phi}{\partial r}-\frac{\phi}{r}>0 .
\end{aligned}
$$

So, we can estimate the left hand side of (6.3) from below as follows.

(6. 12) $\frac{\varepsilon_{0}}{2}\|\mathscr{D} v\|_{\beta, E_{2}}^{2}-C\|\mathscr{D} v\|_{\beta_{1,2}}^{2} \leq$ (the left hand side of $\left.(6.3)\right)$. 
Further for an arbitrary $\varepsilon>0$, the following inequality holds.

$$
\begin{aligned}
I_{1}+I_{2} & \leq C\left(\|\mathscr{D} v\|_{\beta, E_{1}}\|f\|_{\beta+1}+\|v\|_{\beta-1}\|\mathscr{D} v\|_{\beta}\right) \\
& \leq \varepsilon\|\mathscr{D} v\|_{\beta, E_{1}}^{2}+C_{\varepsilon}\left(\|f\|_{\beta+1}^{2}+\|v\|_{\beta-1}^{2}\right) \\
& \leq \varepsilon\|\mathscr{D} v\|_{\beta, E_{2}}^{2}+C_{\varepsilon}\left(\|f\|_{\beta+1}^{2}+\|v\|_{\beta-1}^{2}+\|\mathscr{D} v\|_{B_{1,2}}^{2}\right) .
\end{aligned}
$$

Summing up, we can see by (6.3), (6.12) and (6.13)

$$
\|\mathscr{D} v\|_{\beta, E_{2}}^{2} \leq C\left(\|f\|_{\beta+1}^{2}+\|v\|_{\beta-1}^{2}+\|\mathscr{D} v\|_{B_{1,2}}^{2}\right)+I_{6}+I_{7} .
$$

From (6.11) and $(6.14)$, we can get $(6.10)$.

Q.E.D.

Proposition 6. The following inequality holds:

$$
\left\|D_{v}\right\|_{\beta, E_{1}}^{2} \leq C\left(\|v\|_{\beta-1}^{2}+\|f\|_{\beta+1}^{2}\right),
$$

where $v=e^{i x} u, u \in C_{0}^{\infty}\left(\boldsymbol{R}^{n}\right)$, and the constant $C$ does not depend upon $\kappa \in \bar{K}_{ \pm}$.

Proof. By Schwarz' inequality, we have by Proposition 5,

$$
\left\|D_{v}\right\|_{\beta, E_{1}}^{2} \leq C\left(\|v\|_{\beta-1}^{2}+\|f\|_{\beta+1}^{2}+\|\mathscr{D} v\|_{\beta-\delta / 2, E_{1}}^{2}+\kappa_{2}^{2}\|v\|_{\beta-\delta / 2}^{2}\right) .
$$

Let us recall the inequality in Lemma 2.4 of $\S 2$, that is,

$$
\kappa_{2}^{2}\|v\|_{\beta}^{2} \leq C\|f\|_{\beta+1}^{2} \text {. }
$$

which can be proved without using Lemma 2.2. Hence we have

$$
\|\mathscr{D} v\|_{\beta, E_{1}}^{2} \leq C\left(\|v\|_{\beta-1}^{2}+\|f\|_{\beta+1}^{2}+\|\mathscr{D} v\|_{\beta-\delta / 2, E_{1}}^{2}\right) .
$$

From this, taking $R$ sufficiently large, we have

$$
\|\mathscr{D} v\|_{\beta, E_{1}}^{2} \leq C\left(\|v\|_{\beta-1}^{2}+\|f\|_{\beta+1}^{2}+\|\mathscr{D} v\|_{B_{1}, R}^{2}\right) .
$$

By Ikebe-Saitō ([7], Lemma 2.1), we have

$$
\|\mathscr{D} v\|_{B_{1}, R}^{2} \leq C\left(\|v\|_{\beta-1}^{2}+\|f\|_{\beta+1}^{2}\right) \text {. }
$$

Taking into account of $(6.16)$ and $(6.17)$, we can prove $(6.15)$.

$$
\text { Q.E.D. }
$$

Proof of Lemma 2.2. By Theorem 2 of $\S 2\|v\|_{\beta-1}^{2} \leq C\|f\|_{\beta+1}^{2}$, hence we have the following a-priori estimate

$$
\|\mathscr{D} v\|_{\beta, E_{1}}^{2} \leq C\left\|\left(H-\kappa^{2}\right) u\right\|_{\beta+1}^{2},
$$


for $v=e^{i x} u, u \in C_{0}^{\infty}\left(\boldsymbol{R}^{n}\right) \kappa \in \bar{K}_{ \pm}$.

Extension of (6.18) to the general case can be treated in the same way as Ikebe-Saito [7] using the fact that the set $\left\{\left(H-\kappa^{2}\right) u: u \in C_{0}^{\infty}\left(\boldsymbol{R}^{n}\right)\right\}$ is dense in $L_{2,\left(3-\varepsilon_{0}\right) / 2}$, so we omit the details.

Q.E.D.

Acknowledgement: The author wishes to express his sincere gratitude to Prof. T. Ikebe for his unceasing encouragement and valuable instruction.

\section{References}

[1] Alsholm, P. and Kato, T., Scattering with long-range potentials, Proc. Sympo. Pure Math., 23, 393-399, American Mathematical Society, Providence, R. I., (1973).

[2] Alsholm, P., Wave operators for long-range scattering, Thesis, U. C. Berkeley, (1972).

[3] Ikebe, T., Eigenfunction expansions associated with the Schrödinger operators and their applications to scattering theory, Arch. Rational Mech. Anal., 5 (1960), 1-34.

[4] Ikebe, T., Spectral representations for Schrödinger operators with long-range potentials, J. Functional Anal., 20 (1975), 158-177.

[5] Ikebe, T., Spectral representations for Schrödinger operators with long-range potentials,-Perturbation by short-range potentials-, Publ. RIMS, Kyoto Univ., 11(1976), 551-558.

[6] Ikebe, T., Wave operators and spectral representations, (a lecture given at the Symposium at Katata on Partial Differential Equations, October 1972).

[7] Ikebe, T. and Saitō, Y., Limiting absorption method and absolute continuity for the Schrödinger operator, J. Math. Kyoto Univ., 7 (1972), 513-542.

[8] Kato, T. and Kuroda, S. T., Theory of simple scattering and eigenfunction expansions, Functional analysis and related fields, Springer, 1970, 99-131.

[9] Kato, T. and Kuroda, S. T., Abstract theory of scattering, Rocky Mt. J. Math., 1 (1971), 127-171.

[10] Pinchuk, G., Abstract time-independent wave operator theory for long-range potentials, (to appear).

[11] Saito, Y., On the asymptotic behavior of the solutions of the Schrödinger equation $\left(-\Delta+Q(y)-\kappa^{2}\right) V=F$, (to appear).

[12] Saitō, Y., Eigenfunction expansions for the Schrödinger operators with long-range potentials $Q(y)=O\left(|y|^{-\varepsilon}\right) \varepsilon>0$, (to appear). 